\title{
Intra- and interspecific discrimination of Scorpaena species from the Aegean, Black, Mediterranean and Marmara seas
}

\author{
Serdar Yedier, Derya Bostanci \\ Department of Molecular Biology and Genetics, Faculty of Arts and Sciences, Ordu University, Ordu, Turkey. \\ (SY) (Corresponding author) E-mail: serdar7er@gmail.com. ORCID iD: https://orcid.org/0000-0003-0017-3502 \\ (DB) E-mail: deryabostanci@ gmail.com. ORCID iD: https://orcid.org/0000-0003-3052-9805
}

\begin{abstract}
Summary: This study was conducted to discriminate five Scorpaena species and populations of each species according to morphometric characters. A total of 1865 fish specimens were collected from the eight locations in the four Turkish seas: Antalya, Balıkesir, Çanakkale, Hatay, İzmir, Marmara Ereğlisi, Ordu and Sile. In the study, 26 morphometric traits were measured for intra- and interspecific discrimination of five Scorpaena species. The data were subjected to analysis of variance, principal components analysis (PCA) and canonical discriminant analysis. As results of the PCA, 10 traits for $S$. maderensis and S. scrofa, 12 traits for S. elongata and 13 traits for $S$. notata and S. porcus were found to be important for intraspcific discrimination. The overall classification scores of intraspecific discrimination were determined as $94.6 \%$ for S. elongata, $90.5 \%$ for S. maderensis, $96.7 \%$ for S. notata, $96.5 \%$ for S. porcus and $92.2 \%$ for S. scrofa. The PCA indicated that 13 morphometric measurements among the 26 traits are important in the interspecific discrimination of five Scorpaena species. The cross-validated canonical discriminant analysis was correctly classified as $97.4 \%$ at the Scorpaena species level. The discrimination of correctly classified species ranged from $94.8 \%$ to $100 \%$. Finally, we demonstrated that the morphometric characters examined in the present study can be used successfully in the intra- and interspecific discrimination of Scorpaena species from different habitats.
\end{abstract}

Keywords: Scorpaena; morphometric; intra- and interspecific; discriminant analysis; four seas.

Discriminaciones intra e interespecies: especies de Scorpaena del mar Negro, el mar Egeo, el mar de Mármara y Mediterráneo

Resumen: Este estudio se realizó para discriminar cinco especies de Scorpaena y poblaciones de cada especie en función de los caracteres morfométricos. Se recolectaron un total de 1865 especímenes de peces de los ocho lugares como Antalya, Balıkesir, Çanakkale, Hatay, İzmir, Marmara Ereğlisi, Ordu y Şile en los cuatro mares turcos. En el estudio, se midieron 26 rasgos morfométricos para discriminaciones intra e interespecies de cinco especies de Scorpaena. Los datos se sometieron a ANOVA, análisis de componentes principales (PCA) y análisis discriminante canónico (CDA). Como resultado del PCA, 10 rasgos para $S$. maderensis y $S$. scrofa, 12 rasgos para $S$. elongata, 13 rasgos para $S$. notata y $S$. porcus son importantes para la discriminación intraespecífica. Las puntuaciones generales de clasificación de discriminación intraespecífica se determinaron como $94,6 \%$ para S. elongata, $90,5 \%$ para S. maderensis, $96,7 \%$ para $S$. notata, $96,5 \%$ para $S$. porcus y $92,2 \%$ para S. scrofa. La PCA indicó que 13 medidas morfométricas entre los 26 rasgos son importantes en la discriminación interespecífica de cinco especies de Scorpaena. El análisis discriminante canónico con validación cruzada clasificó correctamente como 97.4\% a nivel de la especie Scorpaena. La discriminación de especies correctamente clasificadas osciló entre 94,8 y 100\%. Finalmente, demostramos que los caracteres morfométricos examinados en el presente estudio se pueden utilizar con éxito en las discriminaciones intra e interespecies de especies de Scorpaena de diferentes hábitats.

Palabras clave: Scorpaena; morfométrico; intra e interespecies; análisis discriminante; cuatro mares.

Citation/Como citar este artículo: Yedier S., Bostanci D. 2021. Intra- and interspecific discrimination of Scorpaena species from the Aegean, Black, Mediterranean and Marmara seas. Sci. Mar. 85(3): 197-209. https://doi.org/10.3989/ scimar.05185.018

Editor: J. Lloret.

Received: March 31, 2021. Accepted: June 1, 2021. Published: August 13, 2021.

Copyright: ( $) 2021$ CSIC. This is an open-access article distributed under the terms of the Creative Commons Attribution 4.0 International (CC BY 4.0) License. 


\section{INTRODUCTION}

There are more than 32000 species of fish, accounting for over half of all vertebrate animals (Nelson et al. 2016). They are dispersed over wide geographic areas, and the environmental conditions can affect traits such as reproduction, fertility and longevity (Rawat et al. 2017). The situations experienced by fish species in their life cycle may also affect their morphometric characteristics. Variations in growth, development and maturation of fish caused by environmental factors cause differences in body shape even within the same genus and species (Cadrin 2000). Information on stock structure, species identification and differentiation is useful for developing management strategies that will help conserve biodiversity associated with species, subspecies, and stocks (Turan et al. 2005, Cadrin et al. 2014). Furthermore, identifying the intra- and interspecific differences/similarities of fish with variable life history characteristics is quite important for understanding population dynamics and evaluating sustainable harvests (Turan et al. 2005, Cadrin et al. 2014). There is also a need to determine how many stocks are managed in a given area and clarify how different stocks are susceptible to fishing pressure and unfavourable environmental conditions (Baldwin et al. 2012). Since genotypic and phenotypic differentiation between fish populations that occurs due to isolation may lead to speciation or the formation of a different population, it is important to examine the degree of differentiation at both the intra- and interspecific levels. Morphometric analyses have been used for inter- and intraspecific identification/distinction of many freshwater and marine fish species. such as Rastrelliger $\mathrm{ka}$ nagurta from peninsular India (Jayasankar et al. 2004), Clarias gariepinus from Turkey (Turan et al. 2005), Pomatomus saltatrix from the Aegean, Black, and Mediterranean seas (Turan et al. 2006), the genus Puntius from Assam, India (Choudhury et al. 2011), Catla catla from India (Ujjainia and Kohli 2011), rattail fish from New Zealand (Ibáñez and Jawad 2018), Barbonymus spp. from Aceh, Indonesia (Batubara et al. 2018) and Macrognathus pancalus from Bangladesh (Mahfuj et al. 2019a).

The family Scorpaenidae includes approximately 23 genera (from 210 to 223 species) distributed both in marine and freshwater waters at medium and great (more than $700 \mathrm{~m}$ ) depths in a variety of aquatic habitats (Froese and Pauly 2020). Scorpaenid systematics are complicated and unsettled (Froese and Pauly 2020). Arculeo and Lo Brutto (2014) and Akalın et al. (2011) indicated that many species of the Scorpaenidae families are quite difficult to define morphologically because small individuals especially are very similar and the characters for describing species are not easy to use. The family includes many fish species that are mostly found in marine waters but rarely spread to freshwaters. One of the popular genera in the family Scorpaenidae is the Scorpaena genus. Currently, six valid species are recognized in this genus from the Turkish coasts; the black scorpionfish (S. porcus Linnaeus, 1758) and the small red scor- pionfish (S. notata Rafinesque, 1810) in the Aegean, Black, Mediterranean and Marmara seas; the slender rockfish (S. elongata Cadenat, 1943), the Madeira rockfish (S. maderensis Valenciennes, 1833) and Cadenat's rockfish (S. loppei Cadenat, 1943) from the Mediterranean Aegean seas; and the red scorpionfish (S. scrofa Linnaeus, 1758) from the Agean, Mediterranean and Marmara seas. Five species belonging to the Scorpaena genus are reported in large numbers in Turkish waters, but $S$. loppei is reported in very few numbers (Keskin and Ery1lmaz 2009).

Many studies have provided detailed information with diagnostic features on the distribution and biology of Scorpaena species (Hureau and Litvinenko 1986, Fischer et al. 1987, Morato et al. 2001). However, no detailed study has been made on the discrimination of Scorpaena species in Turkish marine waters, and only a few studies have been made on Scorpaeniformes species in the Mediterranean Sea. These studies were addressed using cytogenetics (Caputo et al. 1998), meristic characters and genetic analysis of the mitochondrial 16S rDNA gene (Turan et al. 2009). The literature includes a limited number of morphometric studies of the genus Scorpaena, but no morphology-based study in which intra- and interspecific comparisons were made together. The present study was therefore undertaken to investigate the intra- and interspecific discrimination of five Scorpaena species (S. elongata, S. maderensis, S. notata, S. porcus and $S$. scrofa) inhabiting the Aegean, Black, Mediterranean and Marmara seas on the basis of morphometric characters.

\section{MATERIALS AND METHODS}

\section{Ethical statement}

All protocols for fish capture were approved by the Turkish Agricultural Research and Policy General Directorate. The care and use of experimental animals complied with Ordu University Animal Experiments Local Ethics Committee animal welfare laws, guidelines and policies, as approved by the Ordu University Animal Experiments Local Ethics Committee (No:82678388/08).

\section{Sampling}

Fish samples were collected during the 2019-2020 fishing season from the eight locations along the coastline of Turkey's four seas (Fig. 1); İzmir (Aegean Sea), Antalya and Hatay (Mediterranean Sea) for $S$. elongata; Antalya (Mediterranean Sea), Balıkesir and İzmir (Aegean Sea) for S. maderensis; İzmir (Aegean Sea), Hatay (Mediterranean Sea), Marmara Ereğlisi (Sea of Marmara) and Şile (Black Sea) for S. notata; İzmir (Aegean Sea), Hatay (Mediterranean Sea), Marmara Ereğlisi (Sea of Marmara) and Ordu (Black Sea) for S. porcus; and Çanakkale (Sea of Marmara), İzmir (Aegean Sea) and Hatay (Mediterranean Sea) for $S$. scrofa. All samples were preserved and fixed in $70 \%$ ethanol and deposited at Ordu University. 


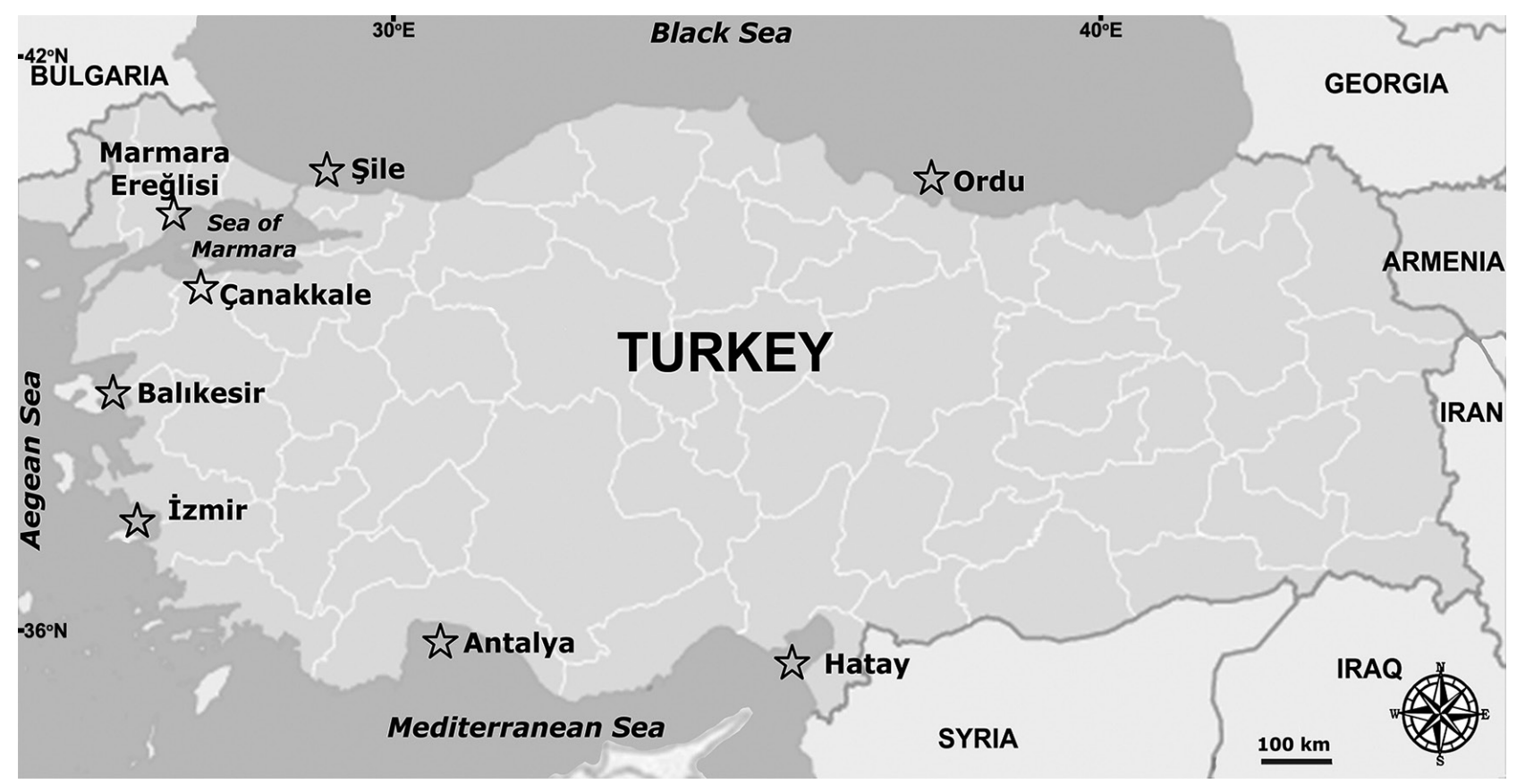

Fig. 1. - Sampling locations of Scorpaena spp. from the Aegean, Black, Mediterranean and Marmara seas.

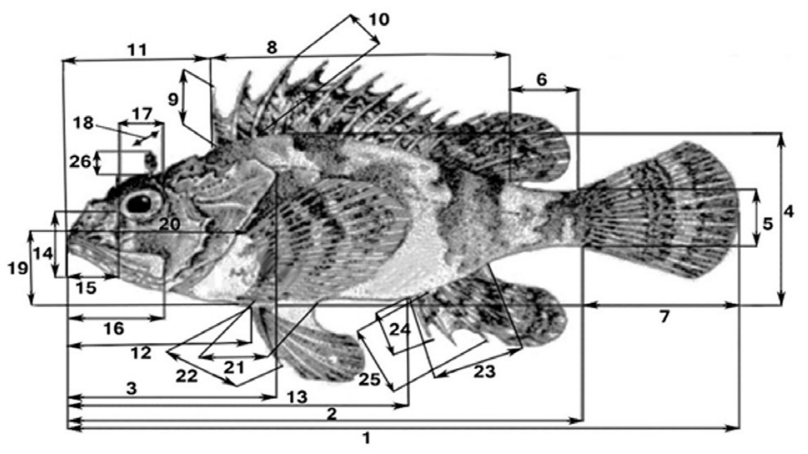

Fig. 2. - Morphometric measurements used in this study on Scorpaena sp. (Ferri et al. 2010, modified).

\section{Morphometric analysis}

A total of 26 metric measurements were used: total length (TL) (1), standard length (SL) (2), head length (HL) (3), body height (BL) (4), caudal peduncle height (CPH) (5), caudal peduncle length (CPL) (6), caudal fin length (CL) (7), dorsal fin base length (DBL) (8), shortest dorsal fin spine length (SDL) (9), longest dorsal fin spine length (LDL) (10), predorsal length (PDL) (11), preventral length (PVL) (12), preanal length (PAL) (13), preorbital height (POH) (14), snout length (NL) (15), maxilla length (ML) (16), eye diameter (ED) (17), interorbital distance (IOD) (18), pectoral fin base length (PBL) (19), prepectoral length (PPL) (20), ventral fin base length (VBL) (21), ventral fin spine length (VSL) (22), anal fin base length (ABL) (23), shortest anal fin spine length (SAL) (24), longest anal fin spine length (LAL) (25) and supraocular tentacle length (STL) (26) (Fig 2). These metric measurements from each individual were taken on the left side of the fish body by the same researcher using a digital caliper $( \pm 0.01 \mathrm{~mm})$ and a millimetre ruler $( \pm 0.1 \mathrm{~cm})$. The sex of each fish sample was determined by internal inspections after the morphometric measurements had been obtained.

\section{Statistical analysis}

The data were tested for normality and homogeneity of variances using the Kolmogorov-Smirnov (K-S) test and the Levene test, respectively. In addition, we investigated whether there was a difference between the data of male and female individuals. An analysis of variance (ANOVA) with Tukey comparisons of morphometric characters was conducted to test for variation among populations and species. Moreover, before running further analysis, the size effects of all morphometric variables were eliminated, as described by Elliott et al. (1995). The equation is as follows: $\mathrm{M}_{\text {adj }}=\mathrm{M}$ $\left(\mathrm{L}_{\mathrm{s}} / \mathrm{L}_{\mathrm{o}}\right)^{\mathrm{b}}$, where $\mathrm{M}$ is the original value of the morphometric measure, $M_{a d j}$ is the adjusted size of the measure, $\mathrm{L}_{0}$ is the standard length of the fish and $\mathrm{L}_{\mathrm{s}}$ is the mean of the standard length of all fish. The parameter b was estimated for each character from the observed data of slope of the regression of $\log \mathrm{M}$ in $\log \mathrm{L}_{\mathrm{o}}$, using all fishes. Size-adjusted data were subjected to principal component analysis (PCA) and canonical discriminant analysis (CDA) to identify intra- and interspecific differences of the five Scorpaena species. Large factor loadings (positive or negative) of PCA indicate that a particular variable has a strong relationship to a particular principal component. Loadings of at least 0.3 magnitude were taken into account when making intraand interspecific distinctions. The UPGMA clustering method was used to generate a dendrogram for intraand interspecific discrimination of the genus Scorpae$n a$ by computing the Euclidian distance values of morphometric measurements. Wilks' lambda $(\lambda)$ was used 
Table 1. - Descriptive statistics (Mean $\pm \mathrm{SD}$ ) and ANOVA results of morphometric measurements of S. elongata populations.

\begin{tabular}{|c|c|c|c|c|c|}
\hline \multirow{2}{*}{$\begin{array}{l}\text { Morphometric } \\
\text { Measurements }\end{array}$} & \multirow{2}{*}{$\begin{array}{l}\text { Antalya } \\
(n=109)\end{array}$} & \multirow{2}{*}{$\begin{array}{l}\text { Hatay } \\
(\mathrm{n}=113)\end{array}$} & \multirow{2}{*}{$\begin{array}{c}\text { İzmir } \\
(\mathrm{n}=110)\end{array}$} & \multicolumn{2}{|c|}{ ANOVA } \\
\hline & & & & $\mathrm{F}$ values & $P$ values \\
\hline $\mathrm{TL}(\mathrm{cm})$ & $15.81 \pm 4.11^{b}$ & $14.10 \pm 2.72^{c}$ & $17.26 \pm 3.05^{\mathrm{a}}$ & 24.98 & 0.001 \\
\hline $\mathrm{SL}(\mathrm{cm})$ & $12.68 \pm 3.43^{\mathrm{b}}$ & $11.23 \pm 2.25^{\mathrm{c}}$ & $13.94 \pm 2.54^{\mathrm{a}}$ & 26.64 & 0.001 \\
\hline $\mathrm{HL}(\mathrm{cm})$ & $5.14 \pm 1.25^{\mathrm{b}}$ & $4.71 \pm 0.94^{c}$ & $5.76 \pm 1.05^{\mathrm{a}}$ & 26.22 & 0.001 \\
\hline $\mathrm{BL}(\mathrm{mm})$ & $41.69 \pm 12.80^{b}$ & $36.30 \pm 8.06^{c}$ & $46.18 \pm 8.10^{\mathrm{a}}$ & 27.93 & 0.001 \\
\hline $\mathrm{CPH}(\mathrm{mm})$ & $12.18 \pm 3.65^{\mathrm{a}}$ & $10.55 \pm 2.04^{b}$ & $12.21 \pm 2.21^{\mathrm{a}}$ & 13.68 & 0.001 \\
\hline CPL (mm) & $11.72 \pm 4.57^{\mathrm{a}}$ & $9.73 \pm 1.59^{\mathrm{b}}$ & $10.13 \pm 2.00^{\mathrm{b}}$ & 13.52 & 0.001 \\
\hline $\mathrm{CL}(\mathrm{cm})$ & $3.13 \pm 0.70^{\mathrm{b}}$ & $2.89 \pm 0.54^{\mathrm{c}}$ & $3.38 \pm 0.57^{\mathrm{a}}$ & 17.86 & 0.001 \\
\hline DBL (cm) & $7.64 \pm 2.05^{\mathrm{b}}$ & $6.80 \pm 1.40^{\mathrm{c}}$ & $8.39 \pm 1.52^{\mathrm{a}}$ & 25.07 & 0.001 \\
\hline $\mathrm{SDL}(\mathrm{mm})$ & $10.78 \pm 1.96^{\mathrm{b}}$ & $10.79 \pm 1.82^{\mathrm{b}}$ & $11.79 \pm 1.36^{\mathrm{a}}$ & 12.31 & 0.001 \\
\hline LDL (mm) & $21.34 \pm 5.11^{\mathrm{b}}$ & $19.22 \pm 3.40^{\mathrm{c}}$ & $23.18 \pm 3.79^{\mathrm{a}}$ & 25.48 & 0.001 \\
\hline PDL (cm) & $3.88 \pm 1.34^{b}$ & $3.70 \pm 0.62^{\mathrm{b}}$ & $4.25 \pm 0.73^{\mathrm{a}}$ & 9.56 & 0.001 \\
\hline PVL (cm) & $5.21 \pm 1.33^{\mathrm{b}}$ & $4.64 \pm 0.91^{\mathrm{c}}$ & $5.66 \pm 1.01^{\mathrm{a}}$ & 24.38 & 0.001 \\
\hline PAL (cm) & $9.05 \pm 2.64^{\mathrm{b}}$ & $8.39 \pm 1.61^{\mathrm{c}}$ & $10.22 \pm 1.87^{\mathrm{a}}$ & 21.96 & 0.001 \\
\hline POH (mm) & $11.30 \pm 2.74^{\mathrm{b}}$ & $10.30 \pm 1.99^{\mathrm{c}}$ & $12.50 \pm 2.30^{\mathrm{a}}$ & 24.26 & 0.001 \\
\hline NL (mm) & $12.42 \pm 3.25^{\mathrm{b}}$ & $11.37 \pm 2.15^{\mathrm{c}}$ & $13.66 \pm 2.31^{\mathrm{a}}$ & 21.54 & 0.001 \\
\hline ML (mm) & $27.13 \pm 6.92^{\mathrm{b}}$ & $24.29 \pm 4.47^{\mathrm{c}}$ & $29.81 \pm 5.21^{\mathrm{a}}$ & 27.04 & 0.001 \\
\hline ED (mm) & $17.84 \pm 3.25^{\mathrm{a}}$ & $17.11 \pm 2.41^{\mathrm{a}}$ & $11.28 \pm 2.15^{\mathrm{b}}$ & 203.03 & 0.001 \\
\hline IOD (mm) & $6.26 \pm 1.14^{\mathrm{a}}$ & $5.34 \pm 1.04^{\mathrm{b}}$ & $6.50 \pm 1.12^{\mathrm{a}}$ & 34.75 & 0.001 \\
\hline PBL (mm) & $15.85 \pm 3.78^{\mathrm{b}}$ & $14.60 \pm 2.51^{\mathrm{c}}$ & $17.34 \pm 2.76^{\mathrm{a}}$ & 22.31 & 0.001 \\
\hline PPL (cm) & $5.02 \pm 1.64^{b}$ & $4.68 \pm 0.93^{\mathrm{b}}$ & $5.69 \pm 1.04^{\mathrm{a}}$ & 19.08 & 0.001 \\
\hline VBL (mm) & $4.67 \pm 1.77^{b}$ & $3.95 \pm 1.25^{\mathrm{c}}$ & $6.38 \pm 1.71^{\mathrm{a}}$ & 68.56 & 0.001 \\
\hline VSL (mm) & $18.77 \pm 3.56^{\mathrm{b}}$ & $18.12 \pm 2.81^{\mathrm{b}}$ & $19.97 \pm 2.69^{\mathrm{a}}$ & 10.64 & 0.001 \\
\hline $\mathrm{ABL}(\mathrm{mm})$ & $17.75 \pm 4.15^{\mathrm{b}}$ & $16.37 \pm 2.79^{\mathrm{c}}$ & $19.77 \pm 3.05^{\mathrm{a}}$ & 28.62 & 0.001 \\
\hline SAL (mm) & $8.04 \pm 1.48^{b}$ & $7.44 \pm 1.10^{\mathrm{c}}$ & $8.74 \pm 1.18^{\mathrm{a}}$ & 29.48 & 0.001 \\
\hline LAL (mm) & $18.82 \pm 3.95^{\mathrm{b}}$ & $17.60 \pm 2.69^{c}$ & $20.49 \pm 2.96^{\mathrm{a}}$ & 22.34 & 0.001 \\
\hline STL (mm) & $2.23 \pm 0.04^{\mathrm{a}}$ & $2.14 \pm 0.03^{\mathrm{a}}$ & $2.22 \pm 0.06^{\mathrm{a}}$ & 1.16 & 0.314 \\
\hline
\end{tabular}

Table 2. - Jackknife classification matrix of the discriminant canonical analysis applied to the three $S$. elongata populations from the coastline of Turkey's two seas.

\begin{tabular}{lccc}
\hline & \multicolumn{3}{c}{ Predicted Group Membership } \\
\hline Population & Antalya & İzmir & Hatay \\
Antalya & $\mathbf{9 1 . 7 ( 1 0 0 )}$ & - & $8.3(9)$ \\
İzmir & - & $\mathbf{1 0 0 . 0}(\mathbf{1 1 0})$ & - \\
Hatay & $8.0(9)$ & & $\mathbf{9 2 . 0}(\mathbf{1 0 4})$ \\
\hline
\end{tabular}

Overall: $94.6 \%$ of original grouped cases correctly classified.

The correct classification percentages and numbers are in bold; the number of individuals is given in parentheses.

to evaluate both intra- and interspecific discrimination performance of the CDA. Interspecific and intraspecific variances, total variances and their percentages of agreement between real and predicted group membership were calculated for both the populations and Scorpaena species. Jackknife cross-validation procedures were used to validate similarities for both the populations and the species. All tests were conducted using the SPSS (V.21.0) and Past (V.2.17c).

\section{RESULTS}

\section{Intraspecific discrimination}

A total of 1865 fish individuals belonging to five species (S. elongata, S. notata, S. maderensis, S. porcus and $S$. scrofa) from the eight locations of the Aegean, Black, Mediterranean and Marmara seas were studied for morphometric analysis. The morphometric variables showed normality ( $\mathrm{P}>0.05$; K-S test) and homogeneous variance $(\mathrm{P}>0.05$; Levene test). There was no statistically significant difference in terms of morphometric data between female and male individuals ( $\mathrm{P}>0.05$; t-test). For this reason, intra- and interspecific discrimination analyses were carried out by evaluating the data of male and female individuals together.

\section{Scorpaena elongata}

A total of 332 S. elongata individuals were sampled from the Antalya, İzmir and Hatay stations in the Aegean and Mediterranean seas. The descriptive analysis of morphometric measurements of $S$. elongata is presented in Table 1. The one-way ANOVA shows significant $(\mathrm{P}<0.05)$ differences in all the morphometric measurements (except for STL) among the S. elongata populations (Table 1). As a result of the PCA, it was determined that 12 morphometric measurements taken from the samples (body height, longest dorsal fin spine length, preanal length, preorbital height, snout length, maxilla length, eye diameter, pectoral fin base length, ventral fin base length, ventral fin spine length, anal fin base length and longest anal fin spine length) are quite important in the intraspecific distinction of the S. elongata. Morphometric ratios were calculated between these important morphometric characters and standard length for each $S$. elongata population (Supplementary Table S1). These morphometric measurements were selected for the CDA. It was determined that the first two functions are important for the CDA analysis performed for the $S$. elongata populations (F1[97.4\%], $\lambda=0.008, \mathrm{P}<0.001 ; \mathrm{F} 2[2.6 \%], \lambda=0.420$, 


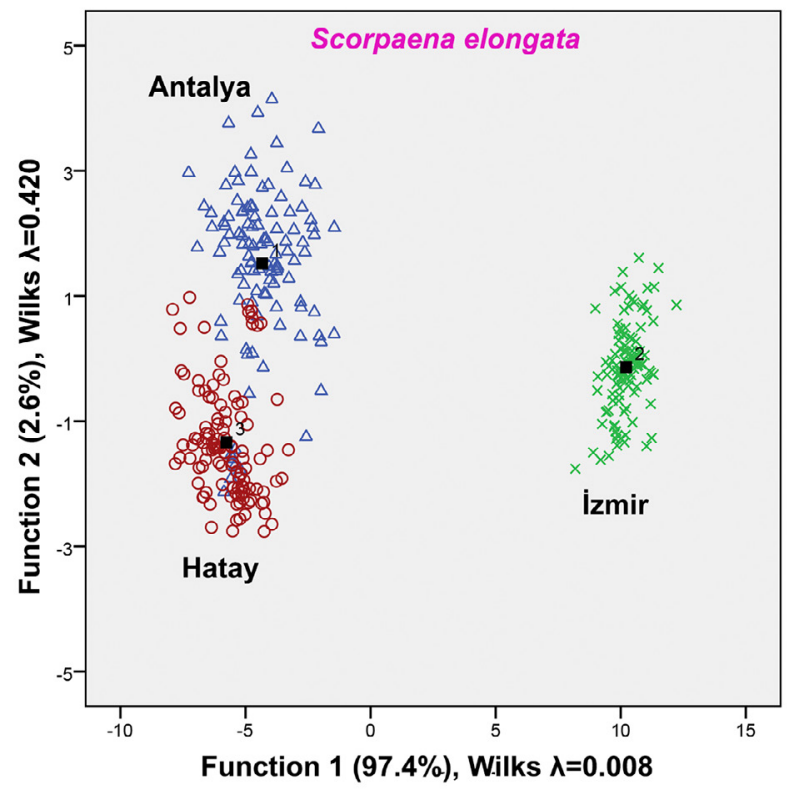

Fig. 3. - Intraspecific discrimination of $S$. elongata using morphometric characters.

$\mathrm{P}<0.001$ ) (Fig 3). It was determined by CDA results that these 12 characters taken from the fish samples were quite effective for discriminating three $S$. elongata populations from each other and that they achieved $94.6 \%$ success in the intraspecific distinction of $S$. elongata (Table 2). S. elongata populations were clustered by hierarchical cluster analyses of meristic data. Antalya and Hatay were the closest $S$. elongata populations and İzmir the most divergent one (Fig. 4).

\section{Scorpaena maderensis}

A total of $326 S$. maderensis individuals were sampled from the Antalya, Balıkesir and Izmir stations in the Aegean and Mediterranean seas. The descriptive analysis of morphometric measurements of $S$. maderensis is presented in Table 3. ANOVA revealed that there were significant $(\mathrm{P}<0.05)$ differences in the TL, SL, HL, BL, CPL, CL, SDL, LDL, PAL, POH, NL, VBL, ABL and LAL measurements among $S$. maderensis populations, and there was no statistically significant $(\mathrm{P}>0.05)$ difference in the CPH, DBL, PDL, PVL, ML, ED, IOD, PBL, PPL, VSL, SAL and STL measurements (Table 3). The PCA analysis indicated that ten morphometric measurements taken from the samples (body height, longest dorsal fin spine length, preanal length, preorbital height, maxilla length, caudal peduncle height, caudal peduncle length, pectoral fin base length, anal fin base length and longest anal fin spine length) are quite important in the intraspecific distinction of S. maderensis. Morphometric ratios were calculated between these important morphometric characters and standard length for each $S$. maderensis population (Supplementary Table S2). These morphometric measurements were selected for the CDA. It was determined that the first two functions were important for the CDA analysis performed for the $S$. maderensis populations

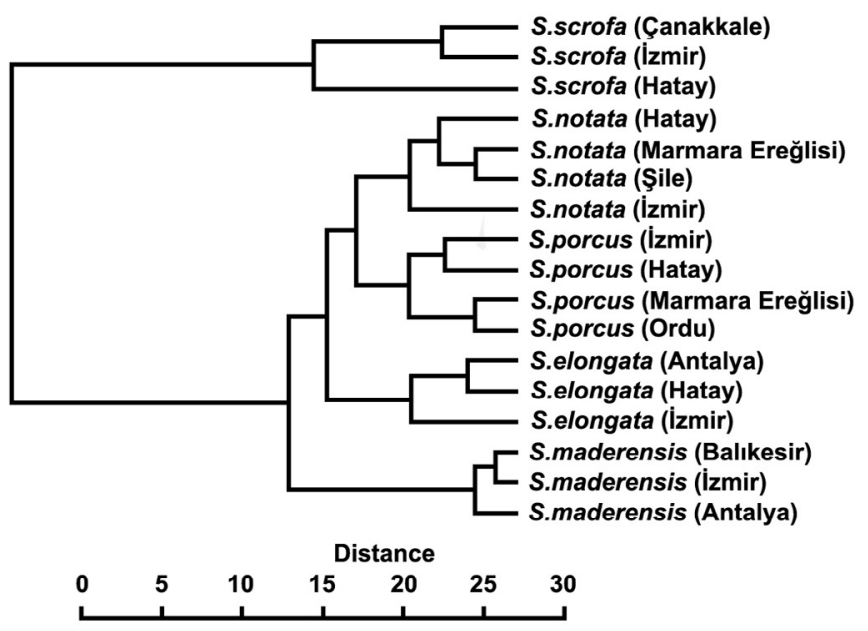

Fig. 4. - The intra- and interspecific dissimilarity of Scorpaena species based on the Euclidian distance of morphometric measurements by hierarchical cluster analysis (UPGMA).

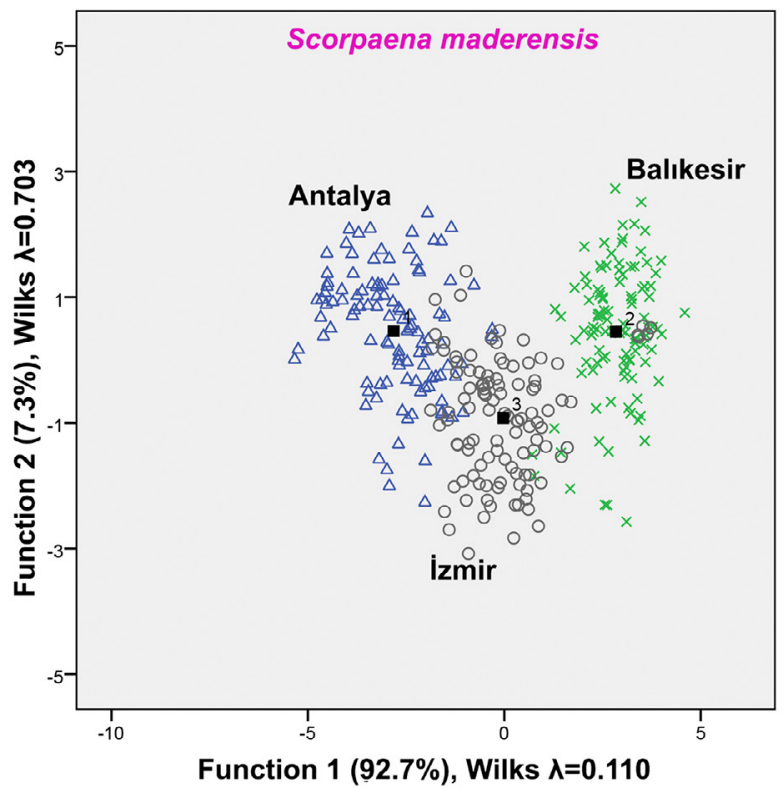

Fig. 5. - Intraspecific discrimination of S. maderensis using morphometric characters.

(F1 [92.7\%], $\lambda=0.110, \mathrm{P}<0.001 ; \mathrm{F} 2[7.3 \%], \lambda=0.703$, $\mathrm{P}<0.001$ ) (Fig 5). The CDA results showed that these 10 characters taken from the fish samples were quite effective for discriminating three $S$. maderensis populations from each other and that they achieved $90.5 \%$ success in the intraspecific distinction of $S$. maderensis (Table 4). S. maderensis populations were clustered by hierarchical cluster analyses of meristic data. Balıkesir and İzmir were the closest $S$. maderensis populations and Antalya the most divergent one (Fig. 4).

\section{Scorpaena notata}

A total of $428 S$. notata individuals were sampled from the İzmir, Hatay, Marmara Ereğlisi and Şile stations in the Aegean, Black, Mediterranean and Mar- 
Table 3. - Descriptive statistics (Mean $\pm \mathrm{SD}$ ) and ANOVA results of morphometric measurements of S. maderensis populations.

\begin{tabular}{|c|c|c|c|c|c|}
\hline \multirow{2}{*}{$\begin{array}{l}\text { Morphometric } \\
\text { Measurements }\end{array}$} & \multirow{2}{*}{$\begin{array}{l}\text { Antalya } \\
(n=109)\end{array}$} & \multirow{2}{*}{$\begin{array}{c}\text { Balıkesir } \\
(n=109)\end{array}$} & \multirow{2}{*}{$\begin{array}{c}\text { İzmir } \\
(\mathrm{n}=108)\end{array}$} & \multicolumn{2}{|c|}{ ANOVA } \\
\hline & & & & $F$ values & $P$ values \\
\hline $\begin{array}{l}\text { TL }(\mathrm{cm}) \\
\text { SL }(\mathrm{cm})\end{array}$ & $\begin{array}{c}12.46 \pm 1.78^{\mathrm{b}} \\
9.55 \pm 1.44^{\mathrm{b}}\end{array}$ & $13.07 \pm 1.52^{\mathrm{a}}$ & $12.69 \pm 1.58^{\mathrm{ab}}$ & 3.82 & 0.023 \\
\hline $\mathrm{SL}(\mathrm{cm})$ & $9.55 \pm 1.44^{\mathrm{b}}$ & $10.03 \pm 1.20^{\mathrm{a}}$ & $9.77 \pm 1.27^{\mathrm{ab}}$ & 3.50 & 0.031 \\
\hline $\mathrm{HL}(\mathrm{cm})$ & $3.98 \pm 0.62^{b}$ & $4.17 \pm 0.50^{\mathrm{a}}$ & $4.05 \pm 0.55^{\mathrm{ab}}$ & 3.18 & 0.043 \\
\hline $\mathrm{BL}(\mathrm{mm})$ & $34.08 \pm 5.11^{\mathrm{b}}$ & $36.27 \pm 4.34^{\mathrm{a}}$ & $34.57 \pm 4.79^{b}$ & 6.32 & 0.002 \\
\hline $\mathrm{CPH}(\mathrm{mm})$ & $10.13 \pm 1.44^{\mathrm{a}}$ & $9.81 \pm 1.23^{\mathrm{a}}$ & $10.18 \pm 1.28^{\mathrm{a}}$ & 2.42 & 0.090 \\
\hline $\mathrm{CPL}(\mathrm{mm})$ & $8.13 \pm 0.97^{\mathrm{a}}$ & $6.48 \pm 0.57^{c}$ & $7.23 \pm 0.92^{b}$ & 104.23 & 0.001 \\
\hline $\mathrm{CL}(\mathrm{cm})$ & $2.91 \pm 0.38^{b}$ & $3.03 \pm 0.32^{\mathrm{a}}$ & $2.94 \pm 0.34^{\mathrm{ab}}$ & 3.61 & 0.028 \\
\hline DBL (cm) & $6.12 \pm 0.95^{\mathrm{a}}$ & $6.38 \pm 0.80^{\mathrm{a}}$ & $6.16 \pm 0.77^{a}$ & 2.91 & 0.056 \\
\hline SDL (mm) & $9.68 \pm 1.42^{\mathrm{a}}$ & $9.34 \pm 1.42^{\mathrm{a}}$ & $8.73 \pm 1.11^{\mathrm{b}}$ & 14.29 & 0.001 \\
\hline LDL (mm) & $16.15 \pm 2.28^{\mathrm{b}}$ & $17.12 \pm 1.78^{\mathrm{a}}$ & $15.66 \pm 1.79^{b}$ & 15.66 & 0.001 \\
\hline PDL (cm) & $2.87 \pm 0.45^{\mathrm{b}}$ & $3.00 \pm 0.37^{\mathrm{a}}$ & $2.93 \pm 0.39^{\mathrm{ab}}$ & 2.99 & 0.052 \\
\hline PVL (cm) & $3.82 \pm 0.53^{\mathrm{a}}$ & $3.88 \pm 0.41^{\mathrm{a}}$ & $3.86 \pm 0.47^{\mathrm{a}}$ & 0.43 & 0.650 \\
\hline PAL (cm) & $6.67 \pm 1.04^{\mathrm{b}}$ & $7.07 \pm 0.90^{\mathrm{a}}$ & $6.77 \pm 0.91^{\mathrm{ab}}$ & 5.16 & 0.006 \\
\hline $\mathrm{POH}(\mathrm{mm})$ & $8.41 \pm 1.31^{\mathrm{b}}$ & $8.84 \pm 1.19^{\mathrm{a}}$ & $8.54 \pm 1.24^{\mathrm{ab}}$ & 3.43 & 0.034 \\
\hline $\mathrm{NL}(\mathrm{mm})$ & $10.79 \pm 1.49^{\mathrm{a}}$ & $10.82 \pm 1.45^{\mathrm{a}}$ & $10.38 \pm 1.19^{\mathrm{a}}$ & 3.36 & 0.036 \\
\hline ML (mm) & $20.32 \pm 3.03^{\mathrm{a}}$ & $21.01 \pm 2.36^{\mathrm{a}}$ & $20.56 \pm 2.64^{\mathrm{a}}$ & 1.87 & 0.156 \\
\hline ED (mm) & $9.23 \pm 1.00^{\mathrm{a}}$ & $9.13 \pm 0.84^{\mathrm{a}}$ & $9.29 \pm 0.84^{\mathrm{a}}$ & 0.87 & 0.421 \\
\hline IOD (mm) & $5.55 \pm 0.65^{\mathrm{a}}$ & $5.56 \pm 0.57^{\mathrm{a}}$ & $5.54 \pm 0.85^{\mathrm{a}}$ & 0.01 & 0.988 \\
\hline PBL (mm) & $13.60 \pm 2.13^{\mathrm{a}}$ & $14.14 \pm 1.77^{\mathrm{a}}$ & $13.68 \pm 1.86^{\mathrm{a}}$ & 2.50 & 0.084 \\
\hline PPL (cm) & $3.64 \pm 0.50^{\mathrm{a}}$ & $3.68 \pm 0.43^{\mathrm{a}}$ & $3.69 \pm 0.45^{\mathrm{a}}$ & 0.47 & 0.626 \\
\hline VBL (mm) & $4.56 \pm 0.57^{\mathrm{a}}$ & $4.44 \pm 0.48^{\mathrm{ab}}$ & $4.38 \pm 0.51^{\mathrm{b}}$ & 3.27 & 0.039 \\
\hline VSL (mm) & $14.85 \pm 1.47^{\mathrm{a}}$ & $14.51 \pm 1.39^{\mathrm{a}}$ & $14.42 \pm 1.25^{\mathrm{a}}$ & 2.87 & 0.058 \\
\hline ABL (mm) & $16.56 \pm 1.96^{\mathrm{b}}$ & $17.31 \pm 1.77^{\mathrm{a}}$ & $16.63 \pm 1.75^{\mathrm{b}}$ & 5.49 & 0.005 \\
\hline SAL (mm) & $9.55 \pm 1.06^{\mathrm{a}}$ & $9.46 \pm 0.99^{\mathrm{a}}$ & $9.35 \pm 0.80^{\mathrm{a}}$ & 1.11 & 0.332 \\
\hline LAL (mm) & $17.24 \pm 1.81^{\mathrm{a}}$ & $17.68 \pm 1.41^{\mathrm{a}}$ & $17.19 \pm 1.51^{\mathrm{a}}$ & 3.07 & 0.048 \\
\hline STL (mm) & $4.40 \pm 0.61^{\mathrm{a}}$ & $4.21 \pm 0.42^{\mathrm{a}}$ & $4.29 \pm 0.49^{\mathrm{a}}$ & 0.02 & 0.979 \\
\hline
\end{tabular}

Table 4. - Jackknife classification matrix of the discriminant canonical analysis applied to the three $S$. maderensis populations from the coastline of Turkey's two seas.

\begin{tabular}{lccc}
\hline & \multicolumn{3}{c}{ Predicted Group Membership } \\
Population & Antalya & Balıesir & İzmir \\
\hline Antalya & $\mathbf{9 0 . 8 ( 9 9 )}$ & - & $9.2(10)$ \\
Balıkesir & - & $\mathbf{9 4 . 5 ( 1 0 3 )}$ & $5.5(6)$ \\
İzmir & $9.3(10)$ & $4.6(5)$ & $\mathbf{8 6 . 1}(\mathbf{9 3 )}$ \\
\hline
\end{tabular}

Overall: $90.5 \%$ of original grouped cases correctly classified.

The correct classification percentages and numbers are in bold; the number of individuals is given in parentheses.

mara seas. The descriptive analysis of morphometric measurements of $S$. notata is presented in Table 5 . ANOVA revealed significant $(\mathrm{P}<0.05)$ differences in all the morphometric measurements among the $S$. notata populations (Table 5). As a result of the PCA, it was determined that 13 morphometric measurements taken from the samples (body height, caudal peduncle height, caudal peduncle length, longest dorsal fin spine length, preorbital height, snout length, maxilla length, eye diameter, pectoral fin base length, anal fin base length, longest anal fin spine length, supraocular tentacle length and ventral fin spine length) are quite important in the intraspecific distinction of S. notata. Morphometric ratios were calculated between these important morphometric characters and standard length for each $S$. notata population (Supplementary Table S3). These morphometric measurements were selected for the CDA. It was determined that the first three functions were important for the CDA analysis performed for the $S$. notata populations (F1[94.7\%], $\lambda=0.003, \quad \mathrm{P}<0.001 ; \mathrm{F} 2[5.2 \%], \quad \lambda=0.200, \quad \mathrm{P}<0.001$; $\mathrm{F} 3[0.1 \%], \lambda=0.947, \mathrm{P}<0.019$ ) (Fig. 6). It was determined from the CDA results that these 13 characters taken from the fish samples were quite effective for discriminating four $S$. notata populations from each other and that they achieved $96.7 \%$ success in the intraspecific distinction of $S$. notata (Table 6). S. notata populations were clustered by hierarchical cluster analyses of meristic data. Marmara Ereğlisi and Şile are the closest populations that were sister populations to the Hatay population. İzmir was the most divergent $S$. notata population (Fig. 4).

\section{Scorpaena porcus}

A total of 459 S. porcus individuals were sampled from the İzmir, Hatay, Marmara Ereğlisi and Ordu stations in the Aegean, Black, Mediterranean and Marmara seas. The descriptive analysis of morphometric measurements of $S$. porcus is presented in Table 7 . The one-way ANOVA showed significant $(\mathrm{P}<0.05)$ differences in all the morphometric measurements among the $S$. porcus populations (Table 7). The PCA analysis indicated that 13 morphometric measurements taken from the samples (body height, caudal peduncle height, caudal peduncle length, shortest dorsal fin spine length, longest dorsal fin spine length, snout length, maxilla length, eye diameter, pectoral fin base length, anal fin base length, longest anal fin spine length, supraocular tentacle length and ventral fin spine length) are quite important for the intraspe- 
Table 5. - Descriptive statistics (Mean $\pm \mathrm{SD}$ ) and ANOVA results of morphometric measurements of S. notata populations.

\begin{tabular}{|c|c|c|c|c|c|c|}
\hline \multirow{2}{*}{$\begin{array}{l}\text { Morphometric } \\
\text { Measurements }\end{array}$} & \multirow{2}{*}{$\begin{array}{c}\text { Hatay } \\
(n=106)\end{array}$} & \multirow{2}{*}{$\begin{array}{c}\text { İzmir } \\
(\mathrm{n}=106)\end{array}$} & \multirow{2}{*}{$\begin{array}{l}\text { Marmara Ereğlisi } \\
\qquad(n=107)\end{array}$} & \multirow{2}{*}{$\begin{array}{c}\text { Şile } \\
(\mathrm{n}=109)\end{array}$} & \multicolumn{2}{|c|}{ ANOVA } \\
\hline & & & & & $F$ values & $\mathrm{P}$ values \\
\hline $\mathrm{TL}(\mathrm{cm})$ & $15.55 \pm 3.58^{b}$ & $18.73 \pm 3.63^{\mathrm{a}}$ & $13.52 \pm 2.59^{c}$ & $13.57 \pm 2.73^{c}$ & 63.86 & 0.001 \\
\hline $\mathrm{SL}(\mathrm{cm})$ & $12.00 \pm 2.84^{\mathrm{b}}$ & $14.46 \pm 2.85^{\mathrm{a}}$ & $10.41 \pm 2.06^{\mathrm{c}}$ & $10.43 \pm 2.19^{c}$ & 61.54 & 0.001 \\
\hline $\mathrm{HL}(\mathrm{cm})$ & $5.10 \pm 1.38^{\mathrm{b}}$ & $6.33 \pm 1.33^{\mathrm{a}}$ & $4.31 \pm 0.92^{\mathrm{c}}$ & $4.36 \pm 0.92^{\mathrm{c}}$ & 71.49 & 0.001 \\
\hline $\mathrm{BL}(\mathrm{mm})$ & $42.08 \pm 8.31^{\mathrm{b}}$ & $48.09 \pm 8.44^{\mathrm{a}}$ & $37.94 \pm 7.68^{\mathrm{c}}$ & $37.77 \pm 7.44^{\mathrm{c}}$ & 39.37 & 0.001 \\
\hline $\mathrm{CPH}(\mathrm{mm})$ & $11.80 \pm 2.98^{\mathrm{b}}$ & $14.66 \pm 3.19^{\mathrm{a}}$ & $10.25 \pm 2.04^{\mathrm{c}}$ & $10.41 \pm 2.28^{\mathrm{c}}$ & 62.60 & 0.001 \\
\hline CPL (mm) & $8.12 \pm 2.59^{b}$ & $10.40 \pm 2.63^{\mathrm{a}}$ & $6.73 \pm 1.18^{\mathrm{c}}$ & $6.86 \pm 1.75^{\mathrm{c}}$ & 77.39 & 0.001 \\
\hline $\mathrm{CL}(\mathrm{cm})$ & $3.56 \pm 0.77^{\mathrm{b}}$ & $4.28 \pm 0.79^{\mathrm{a}}$ & $3.11 \pm 0.55^{\mathrm{c}}$ & $3.15 \pm 0.55^{\mathrm{c}}$ & 69.31 & 0.001 \\
\hline DBL (cm) & $7.38 \pm 1.45^{\mathrm{b}}$ & $8.51 \pm 1.47^{\mathrm{a}}$ & $6.65 \pm 1.34^{c}$ & $6.65 \pm 1.39^{c}$ & 40.68 & 0.001 \\
\hline $\mathrm{SDL}(\mathrm{mm})$ & $10.65 \pm 2.53^{b}$ & $12.67 \pm 2.50^{\mathrm{a}}$ & $9.60 \pm 2.14^{\mathrm{c}}$ & $9.21 \pm 1.55^{\mathrm{c}}$ & 52.43 & 0.001 \\
\hline LDL (mm) & $21.08 \pm 5.98^{b}$ & $27.28 \pm 6.18^{\mathrm{a}}$ & $17.47 \pm 2.96^{\mathrm{c}}$ & $17.47 \pm 2.78^{c}$ & 101.60 & 0.001 \\
\hline PDL (cm) & $3.53 \pm 0.80^{\mathrm{b}}$ & $4.05 \pm 0.73^{\mathrm{a}}$ & $3.04 \pm 0.59^{c}$ & $3.11 \pm 0.68^{\mathrm{c}}$ & 46.82 & 0.001 \\
\hline PVL (cm) & $4.81 \pm 1.33^{\mathrm{b}}$ & $6.11 \pm 1.39^{\mathrm{a}}$ & $4.01 \pm 0.73^{c}$ & $4.04 \pm 0.76^{\mathrm{c}}$ & 85.66 & 0.001 \\
\hline PAL (cm) & $8.53 \pm 2.15^{\mathrm{b}}$ & $10.27 \pm 2.09^{a}$ & $7.28 \pm 1.53^{c}$ & $7.31 \pm 1.65^{\mathrm{c}}$ & 60.23 & 0.001 \\
\hline $\mathrm{POH}(\mathrm{mm})$ & $8.72 \pm 2.41^{\mathrm{b}}$ & $11.65 \pm 3.13^{\mathrm{a}}$ & $7.36 \pm 1.51^{\mathrm{c}}$ & $7.29 \pm 1.57^{\mathrm{c}}$ & 87.37 & 0.001 \\
\hline NL (mm) & $13.84 \pm 4.20^{\mathrm{b}}$ & $17.66 \pm 4.41^{\mathrm{a}}$ & $11.46 \pm 2.42^{\mathrm{c}}$ & $11.42 \pm 2.60^{c}$ & 74.53 & 0.001 \\
\hline $\mathrm{ML}(\mathrm{mm})$ & $25.78 \pm 6.98^{b}$ & $32.05 \pm 7.05^{\mathrm{a}}$ & $21.71 \pm 3.89^{c}$ & $21.97 \pm 4.32^{\mathrm{c}}$ & 75.39 & 0.001 \\
\hline $\mathrm{ED}(\mathrm{mm})$ & $11.17 \pm 3.17^{\mathrm{b}}$ & $14.21 \pm 3.18^{a}$ & $9.31 \pm 1.36^{c}$ & $9.32 \pm 1.45^{\mathrm{c}}$ & 94.87 & 0.001 \\
\hline IOD (mm) & $6.88 \pm 1.96^{\mathrm{b}}$ & $8.78 \pm 1.92^{\mathrm{a}}$ & $5.80 \pm 1.10^{\mathrm{c}}$ & $5.83 \pm 1.22^{\mathrm{c}}$ & 81.98 & 0.001 \\
\hline PBL (mm) & $16.84 \pm 4.13^{b}$ & $20.69 \pm 4.36^{\mathrm{a}}$ & $14.50 \pm 2.86^{\mathrm{c}}$ & $14.58 \pm 3.20^{\mathrm{c}}$ & 66.14 & 0.001 \\
\hline PPL (cm) & $4.49 \pm 1.12^{\mathrm{b}}$ & $5.43 \pm 1.13^{\mathrm{a}}$ & $3.81 \pm 0.71^{\mathrm{c}}$ & $3.84 \pm 0.76^{\mathrm{c}}$ & 67.79 & 0.001 \\
\hline VBL (mm) & $5.30 \pm 1.17^{\mathrm{b}}$ & $6.04 \pm 1.12^{\mathrm{a}}$ & $4.61 \pm 0.85^{\mathrm{c}}$ & $4.62 \pm 0.93^{c}$ & 47.36 & 0.001 \\
\hline VSL (mm) & $17.71 \pm 4.41^{\mathrm{b}}$ & $21.63 \pm 4.35^{\mathrm{a}}$ & $14.62 \pm 2.17^{\mathrm{c}}$ & $14.78 \pm 2.17^{\mathrm{c}}$ & 97.08 & 0.001 \\
\hline $\mathrm{ABL}(\mathrm{mm})$ & $19.29 \pm 2.99^{b}$ & $21.11 \pm 2.88^{\mathrm{a}}$ & $17.61 \pm 2.67^{c}$ & $17.63 \pm 2.86^{c}$ & 36.40 & 0.001 \\
\hline SAL (mm) & $10.64 \pm 1.94^{\mathrm{b}}$ & $11.69 \pm 1.72^{\mathrm{a}}$ & $9.59 \pm 1.61^{\mathrm{c}}$ & $9.77 \pm 1.67^{\mathrm{c}}$ & 32.81 & 0.001 \\
\hline LAL (mm) & $20.17 \pm 3.78^{b}$ & $22.59 \pm 3.16^{\mathrm{a}}$ & $17.97 \pm 2.31^{\mathrm{c}}$ & $18.21 \pm 2.52^{c}$ & 54.70 & 0.001 \\
\hline STL (mm) & $3.15 \pm 0.99^{c}$ & $6.67 \pm 1.64^{\mathrm{a}}$ & $4.27 \pm 0.69^{b}$ & $2.69 \pm 0.58^{\mathrm{d}}$ & 51.56 & 0.001 \\
\hline
\end{tabular}

Table 6. - Jackknife classification matrix of the discriminant canonical analysis applied to the four $S$. notata populations from the coastline of Turkey's four seas.

\begin{tabular}{lcccc}
\hline & \multicolumn{4}{c}{ Predicted Group Membership } \\
Population & İzmir & Hatay & $\begin{array}{c}\text { Marmara } \\
\text { Ereğlisi }\end{array}$ & Şile \\
\hline İzmir & $\mathbf{1 0 0 . 0}(\mathbf{1 0 6})$ & - & - & - \\
Hatay & - & $\mathbf{1 0 0 . 0}(\mathbf{1 0 6})$ & - & - \\
Marmara & - & - & $\mathbf{9 3 . 5}(\mathbf{1 0 0})$ & $6.5(7)$ \\
Ereğlisi & - & - & $6.4(7)$ & $\mathbf{9 3 . 6}(\mathbf{1 0 2})$ \\
Şile & - & - & \\
\hline Overall: 96.7\% of original grouped cases correctly classified. \\
\hline The correct classification percentages and numbers are in bold; the \\
number of individuals is given in parentheses.
\end{tabular}

cific distinction of the $S$. porcus. Morphometric ratios were calculated between these important morphometric characters and standard length for each $S$. porcus population (Supplementary Table S4). These morphometric measurements were selected for the CDA. It was determined that the first three functions are important for the CDA analysis performed for the $S$. porcus populations (F1[90.2\%], $\lambda=0.002, \mathrm{P}<0.001$; $\mathrm{F} 2[5.6 \%], \quad \lambda=0.090, \mathrm{P}<0.001 ; \mathrm{F} 3[4.2 \%], \lambda=0.330$, $\mathrm{P}<0.001$ ) (Fig. 7). It was determined from the CDA results that these 13 characters taken from the fish samples were quite effective for discriminating four $S$. porcus populations from each other and that they achieved $96.5 \%$ success in the intraspecific distinction of $S$. porcus (Table 8). S. porcus populations were clustered by hierarchical cluster analyses of meristic data. Two branches were produced by UPGMA: the first was made up of İzmir and Hatay populations; the

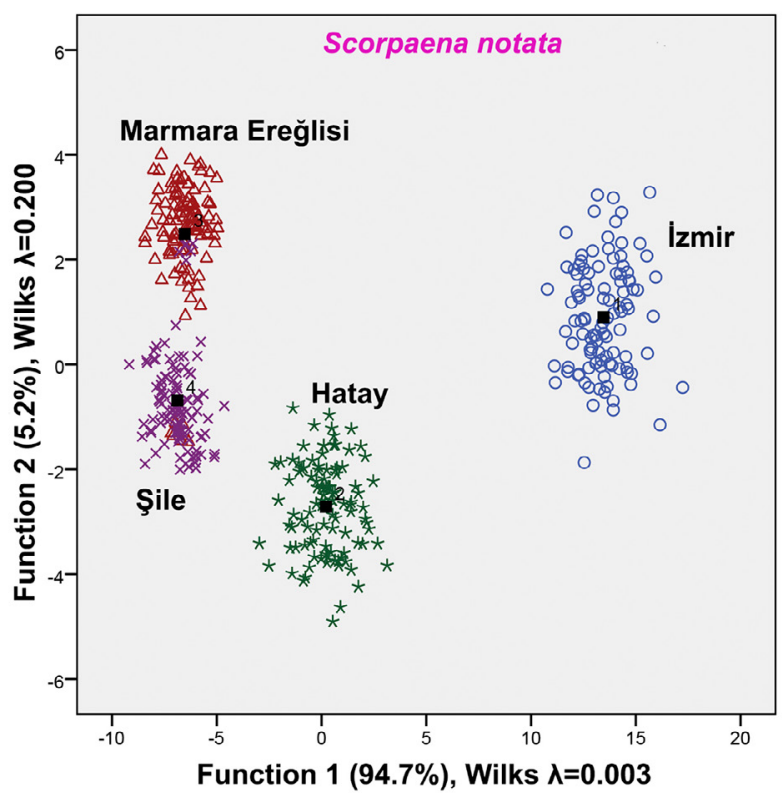

Fig. 6. - Intraspecific discrimination of $S$. notata using morphometric characters.

second was made up of Marmara Ereğlisi and Şile populations. These were the closest $S$. porcus populations (Fig.4).

\section{Scorpaena scrofa}

A total of $320 \mathrm{~S}$. scrofa individuals were sampled from the Çanakkale, İzmir and Hatay stations in the 
Table 7. - Descriptive statistics (Mean $\pm \mathrm{SD}$ ) and ANOVA results of morphometric measurements of S. porcus populations.

\begin{tabular}{|c|c|c|c|c|c|c|}
\hline \multirow{2}{*}{$\begin{array}{l}\text { Morphometric } \\
\text { Measurements }\end{array}$} & \multirow{2}{*}{$\begin{array}{l}\text { Hatay } \\
(n=114)\end{array}$} & \multirow{2}{*}{$\begin{array}{c}\text { İzmir } \\
(\mathrm{n}=115)\end{array}$} & \multirow{2}{*}{$\begin{array}{l}\text { Marmara Ereğlisi } \\
(\mathrm{n}=115)\end{array}$} & \multirow{2}{*}{$\begin{array}{c}\text { Ordu } \\
(\mathrm{n}=115)\end{array}$} & \multicolumn{2}{|c|}{ ANOVA } \\
\hline & & & & & $F$ values & P values \\
\hline TL (cm) & $16.55 \pm 3.98^{b}$ & $18.56 \pm 4.27^{\mathrm{a}}$ & $14.56 \pm 3.82^{\mathrm{c}}$ & $13.94 \pm 4.15^{\mathrm{c}}$ & 30.85 & 0.001 \\
\hline $\mathrm{SL}(\mathrm{cm})$ & $12.85 \pm 3.28^{\mathrm{b}}$ & $14.56 \pm 3.43^{\mathrm{a}}$ & $11.81 \pm 3.12^{\mathrm{bc}}$ & $10.78 \pm 3.43^{\mathrm{c}}$ & 27.42 & 0.001 \\
\hline $\mathrm{HL}(\mathrm{cm})$ & $5.26 \pm 1.31^{\mathrm{b}}$ & $6.31 \pm 1.53^{\mathrm{a}}$ & $5.02 \pm 1.36^{\mathrm{b}}$ & $4.52 \pm 1.52^{\mathrm{c}}$ & 31.99 & 0.001 \\
\hline $\mathrm{BL}(\mathrm{mm})$ & $48.48 \pm 12.37^{b}$ & $63.53 \pm 15.88^{\mathrm{a}}$ & $45.04 \pm 12.55^{\mathrm{b}}$ & $39.18 \pm 12.74^{c}$ & 69.06 & 0.001 \\
\hline CPH (mm) & $13.42 \pm 4.01^{\mathrm{b}}$ & $14.92 \pm 3.72^{\mathrm{a}}$ & $11.59 \pm 3.29^{\mathrm{c}}$ & $10.67 \pm 3.47^{\mathrm{c}}$ & 31.62 & 0.001 \\
\hline CPL (mm) & $10.50 \pm 2.37^{\mathrm{b}}$ & $13.94 \pm 3.71^{\mathrm{a}}$ & $9.42 \pm 2.63^{\mathrm{c}}$ & $8.80 \pm 2.63^{c}$ & 73.52 & 0.001 \\
\hline CL $(\mathrm{cm})$ & $3.70 \pm 0.73^{b}$ & $4.02 \pm 0.85^{\mathrm{a}}$ & $2.74 \pm 0.73^{\mathrm{d}}$ & $3.23 \pm 0.80^{c}$ & 58.89 & 0.001 \\
\hline DBL (cm) & $8.14 \pm 2.04^{b}$ & $8.94 \pm 2.04^{\mathrm{a}}$ & $6.98 \pm 1.83^{\mathrm{c}}$ & $6.81 \pm 1.99^{c}$ & 30.12 & 0.001 \\
\hline $\mathrm{SDL}(\mathrm{mm})$ & $12.31 \pm 3.43^{\mathrm{b}}$ & $13.87 \pm 3.05^{\mathrm{a}}$ & $10.36 \pm 2.80^{\mathrm{c}}$ & $9.76 \pm 3.10^{c}$ & 42.46 & 0.001 \\
\hline LDL (mm) & $19.53 \pm 3.85^{\mathrm{b}}$ & $23.46 \pm 4.56^{\mathrm{a}}$ & $17.58 \pm 4.81^{\mathrm{c}}$ & $17.85 \pm 4.31^{\mathrm{c}}$ & 44.02 & 0.001 \\
\hline PDL (cm) & $3.94 \pm 1.07^{\mathrm{b}}$ & $4.62 \pm 1.15^{\mathrm{a}}$ & $3.68 \pm 1.01^{\mathrm{b}}$ & $3.25 \pm 1.21^{\mathrm{c}}$ & 30.94 & 0.001 \\
\hline PVL (cm) & $5.16 \pm 1.34^{b}$ & $5.61 \pm 1.31^{\mathrm{a}}$ & $4.45 \pm 1.23^{\mathrm{c}}$ & $4.07 \pm 1.24^{\mathrm{c}}$ & 33.92 & 0.001 \\
\hline PAL (cm) & $9.24 \pm 2.38^{b}$ & $10.94 \pm 2.78^{\mathrm{a}}$ & $8.67 \pm 2.33^{b}$ & $7.71 \pm 2.72^{\mathrm{c}}$ & 32.35 & 0.001 \\
\hline $\mathrm{POH}(\mathrm{mm})$ & $9.19 \pm 2.45^{\mathrm{b}}$ & $10.07 \pm 2.41^{\mathrm{a}}$ & $7.29 \pm 1.92^{\mathrm{c}}$ & $6.97 \pm 1.94^{\mathrm{c}}$ & 53.72 & 0.001 \\
\hline NL (mm) & $13.84 \pm 3.28^{\mathrm{b}}$ & $15.94 \pm 3.91^{\mathrm{a}}$ & $12.45 \pm 3.32^{\mathrm{c}}$ & $11.65 \pm 3.71^{\mathrm{c}}$ & 32.19 & 0.001 \\
\hline ML (mm) & $27.16 \pm 6.48^{\mathrm{b}}$ & $31.13 \pm 7.42^{\mathrm{a}}$ & $24.24 \pm 6.43^{\mathrm{c}}$ & $23.02 \pm 7.17^{\mathrm{c}}$ & 31.77 & 0.001 \\
\hline $\mathrm{ED}(\mathrm{mm})$ & $11.00 \pm 1.91^{\mathrm{b}}$ & $15.64 \pm 3.64^{\mathrm{a}}$ & $8.86 \pm 2.44^{\mathrm{d}}$ & $9.85 \pm 2.26^{\mathrm{c}}$ & 148.49 & 0.001 \\
\hline IOD (mm) & $6.61 \pm 1.69^{\mathrm{b}}$ & $9.32 \pm 2.22^{\mathrm{a}}$ & $6.10 \pm 1.64^{\mathrm{bc}}$ & $5.77 \pm 1.75^{\mathrm{c}}$ & 89.70 & 0.001 \\
\hline PBL (mm) & $17.64 \pm 4.27^{\mathrm{b}}$ & $22.19 \pm 5.31^{\mathrm{a}}$ & $18.65 \pm 5.06^{\mathrm{b}}$ & $15.75 \pm 5.52^{\mathrm{c}}$ & 33.14 & 0.001 \\
\hline PPL (cm) & $4.88 \pm 1.33^{\mathrm{a}}$ & $5.19 \pm 1.26^{\mathrm{a}}$ & $4.07 \pm 1.11^{\mathrm{b}}$ & $3.82 \pm 1.15^{\mathrm{b}}$ & 33.00 & 0.001 \\
\hline VBL (mm) & $5.63 \pm 1.21^{\mathrm{a}}$ & $5.22 \pm 1.47^{\mathrm{a}}$ & $5.50 \pm 1.63^{\mathrm{a}}$ & $4.61 \pm 0.88^{b}$ & 13.59 & 0.001 \\
\hline VSL (mm) & $17.92 \pm 3.92^{\mathrm{b}}$ & $22.27 \pm 4.52^{\mathrm{a}}$ & $17.56 \pm 4.71^{\mathrm{b}}$ & $15.38 \pm 4.18^{\mathrm{c}}$ & 51.32 & 0.001 \\
\hline $\mathrm{ABL}(\mathrm{mm})$ & $20.46 \pm 4.49^{\mathrm{b}}$ & $22.89 \pm 4.17^{\mathrm{a}}$ & $17.58 \pm 4.63^{\mathrm{c}}$ & $17.54 \pm 4.45^{\mathrm{c}}$ & 39.06 & 0.001 \\
\hline SAL (mm) & $11.34 \pm 2.49^{b}$ & $13.08 \pm 2.61^{\mathrm{a}}$ & $9.81 \pm 2.62^{\mathrm{c}}$ & $9.65 \pm 2.54^{\mathrm{c}}$ & 45.15 & 0.001 \\
\hline LAL (mm) & $20.02 \pm 3.47^{b}$ & $21.71 \pm 3.32^{\mathrm{a}}$ & $16.71 \pm 4.74^{\mathrm{d}}$ & $18.28 \pm 3.70^{c}$ & 36.44 & 0.001 \\
\hline STL (mm) & $10.93 \pm 1.95^{\mathrm{b}}$ & $15.46 \pm 3.62^{\mathrm{a}}$ & $8.86 \pm 2.43^{\mathrm{d}}$ & $9.81 \pm 2.33^{\mathrm{c}}$ & 1380.04 & 0.001 \\
\hline
\end{tabular}

Table 8. - Jackknife classification matrix of the discriminant canonical analysis applied to the four $S$. porcus populations from the coastline of Turkey's four seas.

\begin{tabular}{lcccc}
\hline Population & İzmir & Hatay & $\begin{array}{c}\text { Marmara } \\
\text { Ereğlisi }\end{array}$ & Ordu \\
\hline İzmir & $\mathbf{1 0 0 . 0}(\mathbf{1 1 5})$ & - & - & - \\
Hatay & - & $\mathbf{1 0 0 . 0}(\mathbf{1 1 4})$ & - & - \\
Marmara & - & - & $\mathbf{9 3 . 0}(\mathbf{1 0 7})$ & $7.0(8)$ \\
Ereğlisi & - & - & $7.0(8)$ & $\mathbf{9 3 . 0}(\mathbf{1 0 7})$ \\
Ordu & - & - &
\end{tabular}

Overall: $96.5 \%$ of original grouped cases correctly classified.

The correct classifications percentages and numbers are in bold; the number of individuals is given in parentheses.

Aegean, Mediterranean and Marmara seas. The descriptive analysis of morphometric measurements of $S$. scrofa is presented in Table 9. ANOVA revealed significant $(\mathrm{P}<0.05)$ differences in all the morphometric measurements (except for PPL and STL) among the $S$. scrofa populations (Table 9). As a result of the PCA, it was determined that ten morphometric measurements taken from the samples (body height, caudal peduncle height, shortest dorsal fin spine length, longest dorsal fin spine length, preorbital height, snout length, maxilla length, pectoral fin base length, ventral fin spine length and anal fin base length) are quite important in the intraspecific distinction of $S$. scrofa. Morphometric ratios were calculated between these important morphometric characters and standard length for each $S$. porcus population (Supplementary Table S5). These morphometric measurements were selected for the CDA. It was deter-

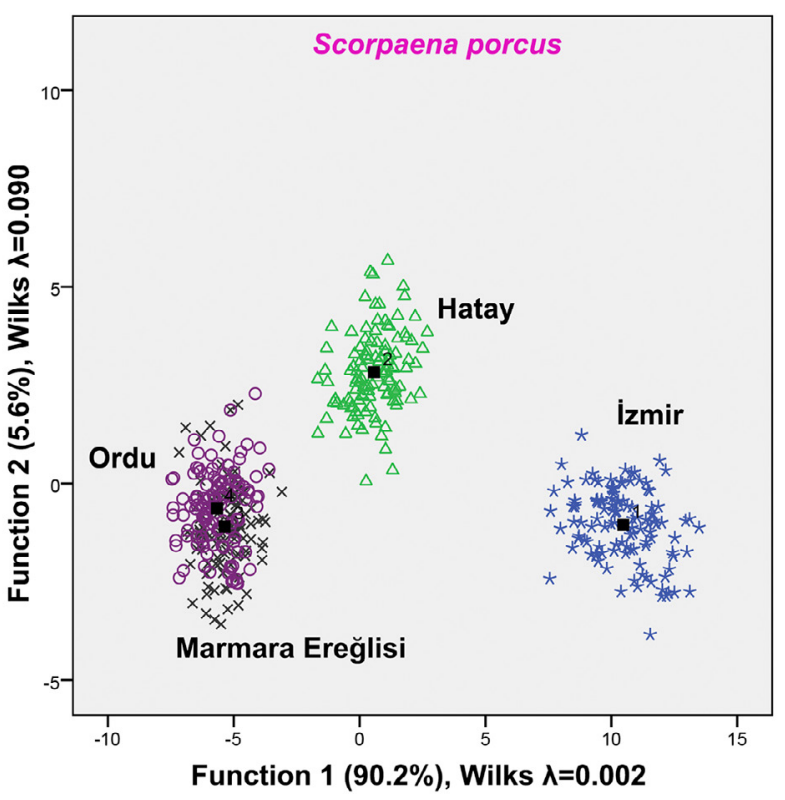

Fig. 7. - Intraspecific discrimination of S. porcus using morphometric characters.

mined that the first two functions were important for the CDA analysis performed for the $S$. scrofa populations (F1[93.7\%], $\lambda=0.055, \mathrm{P}<0.001 ; \mathrm{F} 2[6.3 \%], \lambda=0.600$, $\mathrm{P}<0.001$ ) (Fig. 8). It was determined from the CDA results that these 10 characters taken from the fish samples were quite effective for discriminating four S. scrofa populations from each other and that they achieved $92.2 \%$ success in the intraspecific distinction of $S$. scrofa 
Table 9. - Descriptive statistics (Mean $\pm \mathrm{SD}$ ) and ANOVA results of morphometric measurements of S. scrofa populations.

\begin{tabular}{|c|c|c|c|c|c|}
\hline \multirow{2}{*}{$\begin{array}{l}\text { Morphometric } \\
\text { Measurements }\end{array}$} & \multirow{2}{*}{$\begin{array}{c}\text { Çanakkale } \\
(\mathrm{n}=107)\end{array}$} & \multirow{2}{*}{$\begin{array}{l}\text { Hatay } \\
(\mathrm{n}=107)\end{array}$} & \multirow{2}{*}{$\begin{array}{c}\text { İzmir } \\
(\mathrm{n}=106)\end{array}$} & \multicolumn{2}{|c|}{ ANOVA } \\
\hline & & & & F values & $\mathrm{P}$ values \\
\hline TL (cm) & $22.88 \pm 5.03^{\mathrm{a}}$ & $19.90 \pm 5.63^{\mathrm{b}}$ & $21.57 \pm 3.91^{\mathrm{a}}$ & 9.90 & 0.001 \\
\hline $\mathrm{SL}(\mathrm{cm})$ & $17.71 \pm 3.77^{\mathrm{a}}$ & $15.59 \pm 4.29^{\mathrm{b}}$ & $16.71 \pm 3.01^{\mathrm{ab}}$ & 8.60 & 0.001 \\
\hline $\mathrm{HL}(\mathrm{cm})$ & $7.74 \pm 1.79^{\mathrm{a}}$ & $6.65 \pm 1.98^{\mathrm{b}}$ & $7.51 \pm 1.43^{\mathrm{a}}$ & 11.63 & 0.001 \\
\hline $\mathrm{BL}(\mathrm{mm})$ & $57.99 \pm 12.96^{\mathrm{a}}$ & $50.75 \pm 14.27^{b}$ & $56.04 \pm 10.77^{\mathrm{a}}$ & 9.22 & 0.001 \\
\hline CPH (mm) & $18.22 \pm 4.46^{\mathrm{a}}$ & $15.42 \pm 4.73^{\mathrm{b}}$ & $17.47 \pm 3.34^{\mathrm{a}}$ & 12.59 & 0.001 \\
\hline CPL (mm) & $14.28 \pm 3.06^{\mathrm{a}}$ & $12.64 \pm 3.45^{\mathrm{b}}$ & $13.16 \pm 2.59^{\mathrm{b}}$ & 8.14 & 0.001 \\
\hline CL (cm) & $5.18 \pm 1.31^{\mathrm{a}}$ & $4.31 \pm 1.38^{b}$ & $4.86 \pm 0.95^{\mathrm{a}}$ & 13.62 & 0.001 \\
\hline DBL (cm) & $10.33 \pm 2.06^{\mathrm{a}}$ & $9.39 \pm 2.62^{b}$ & $9.87 \pm 1.71^{\mathrm{ab}}$ & 5.10 & 0.007 \\
\hline SDL (mm) & $16.10 \pm 3.35^{\mathrm{a}}$ & $12.98 \pm 2.68^{\mathrm{c}}$ & $14.78 \pm 2.60^{\mathrm{b}}$ & 31.43 & 0.001 \\
\hline LDL (mm) & $33.28 \pm 8.33^{\mathrm{a}}$ & $27.04 \pm 7.67^{\mathrm{c}}$ & $30.66 \pm 5.87^{b}$ & 19.36 & 0.001 \\
\hline PDL (cm) & $5.86 \pm 1.37^{\mathrm{a}}$ & $5.19 \pm 1.59^{\mathrm{b}}$ & $5.83 \pm 1.09^{\mathrm{a}}$ & 8.11 & 0.001 \\
\hline PVL (cm) & $7.32 \pm 1.65^{\mathrm{a}}$ & $6.42 \pm 1.86^{\mathrm{b}}$ & $6.97 \pm 1.32^{\mathrm{a}}$ & 8.21 & 0.001 \\
\hline PAL (cm) & $13.58 \pm 3.06^{\mathrm{a}}$ & $11.78 \pm 3.36^{\mathrm{b}}$ & $12.85 \pm 2.39^{\mathrm{a}}$ & 9.91 & 0.001 \\
\hline $\mathrm{POH}(\mathrm{mm})$ & $22.97 \pm 5.26^{\mathrm{a}}$ & $19.68 \pm 5.83^{\mathrm{b}}$ & $22.41 \pm 4.21^{\mathrm{a}}$ & 12.44 & 0.001 \\
\hline NL (mm) & $21.80 \pm 6.04^{\mathrm{a}}$ & $17.58 \pm 6.44^{\mathrm{b}}$ & $21.42 \pm 3.98^{\mathrm{a}}$ & 18.59 & 0.001 \\
\hline ML (mm) & $39.55 \pm 8.75^{\mathrm{a}}$ & $34.12 \pm 9.56^{\mathrm{b}}$ & $37.42 \pm 7.03 a$ & 11.08 & 0.001 \\
\hline $\mathrm{ED}(\mathrm{mm})$ & $17.57 \pm 2.14^{\mathrm{a}}$ & $16.75 \pm 1.99^{b}$ & $17.13 \pm 1.84^{\mathrm{ab}}$ & 4.55 & 0.011 \\
\hline IOD (mm) & $10.42 \pm 2.79^{\mathrm{a}}$ & $8.33 \pm 2.91^{\mathrm{b}}$ & $10.34 \pm 2.01^{\mathrm{a}}$ & 22.32 & 0.001 \\
\hline PBL (mm) & $25.09 \pm 6.08^{\mathrm{a}}$ & $20.56 \pm 6.21^{\mathrm{b}}$ & $24.69 \pm 4.12^{\mathrm{a}}$ & 21.60 & 0.001 \\
\hline PPL (cm) & $6.60 \pm 1.47^{\mathrm{a}}$ & $6.59 \pm 1.91^{\mathrm{a}}$ & $6.26 \pm 1.19^{\mathrm{a}}$ & 1.67 & 0.191 \\
\hline VBL (mm) & $7.80 \pm 1.88^{\mathrm{a}}$ & $6.14 \pm 2.13^{\mathrm{b}}$ & $7.30 \pm 1.73^{\mathrm{a}}$ & 20.94 & 0.001 \\
\hline VSL (mm) & $25.52 \pm 4.60^{\mathrm{a}}$ & $22.44 \pm 5.09^{\mathrm{b}}$ & $25.35 \pm 3.25^{\mathrm{a}}$ & 16.70 & 0.001 \\
\hline $\mathrm{ABL}(\mathrm{mm})$ & $24.50 \pm 5.10^{\mathrm{a}}$ & $21.76 \pm 5.55^{\mathrm{b}}$ & $23.31 \pm 3.41^{\mathrm{a}}$ & 8.84 & 0.001 \\
\hline SAL (mm) & $13.01 \pm 3.11^{\mathrm{a}}$ & $10.94 \pm 3.43^{\mathrm{b}}$ & $12.44 \pm 1.38^{\mathrm{a}}$ & 15.67 & 0.001 \\
\hline LAL (mm) & $24.39 \pm 4.49^{\mathrm{a}}$ & $22.34 \pm 5.30^{\mathrm{b}}$ & $23.81 \pm 2.53^{\mathrm{a}}$ & 6.52 & 0.002 \\
\hline $\mathrm{STL}(\mathrm{mm})$ & $1.72 \pm 0.32^{\mathrm{a}}$ & $1.68 \pm 0.20^{\mathrm{a}}$ & $1.71 \pm 0.18^{\mathrm{a}}$ & 0.99 & 0.374 \\
\hline
\end{tabular}

Table 10. - Jackknife classification matrix of the discriminant canonical analysis applied to the four $S$. scrofa populations from the coastline of Turkey's three seas.

\begin{tabular}{lccc}
\hline & \multicolumn{3}{c}{ Predicted Group Membership } \\
Population & Çanakkale & İzmir & Hatay \\
\hline Çanakkale & $\mathbf{8 7 . 9 ( 9 4 )}$ & $12.1(13)$ & - \\
İzmir & $10.4(11)$ & $\mathbf{8 9 . 6 ( 9 5 )}$ & - \\
Hatay & - & $0.9(1)$ & $\mathbf{9 9 . 1}(\mathbf{1 0 6})$ \\
\hline
\end{tabular}

Overall: $92.2 \%$ of original grouped cases correctly classified.

The correct classification percentages and numbers are in bold; the number of individuals is given in parentheses.

(Table 10). S. scrofa populations were clustered by hierarchical cluster analyses of meristic data. Çanakkale and İzmir were the closest $S$. scrofa populations and Hatay the most divergent (Fig. 4).

\section{Interspecific discrimination}

A total of 1865 individuals belonging to five Scorpaena species were sampled from the Antalya, Balıkesir, Çanakkale, İzmir, Hatay, Marmara Ereğlisi, Ordu and Şile stations in the Aegean, Black, Mediterranean and Marmara seas. The descriptive analysis of morphometric measurements of five Scorpaena species is presented in Table 11. The one-way ANOVA showed significant $(\mathrm{P}<0.05)$ differences in all the morphometric measurements among the Scorpaena species (Table 11). The PCA analysis indicated that 13 morphometric measurements taken from the samples (body height, caudal peduncle height, caudal peduncle length, longest dorsal fin spine length, preorbit-

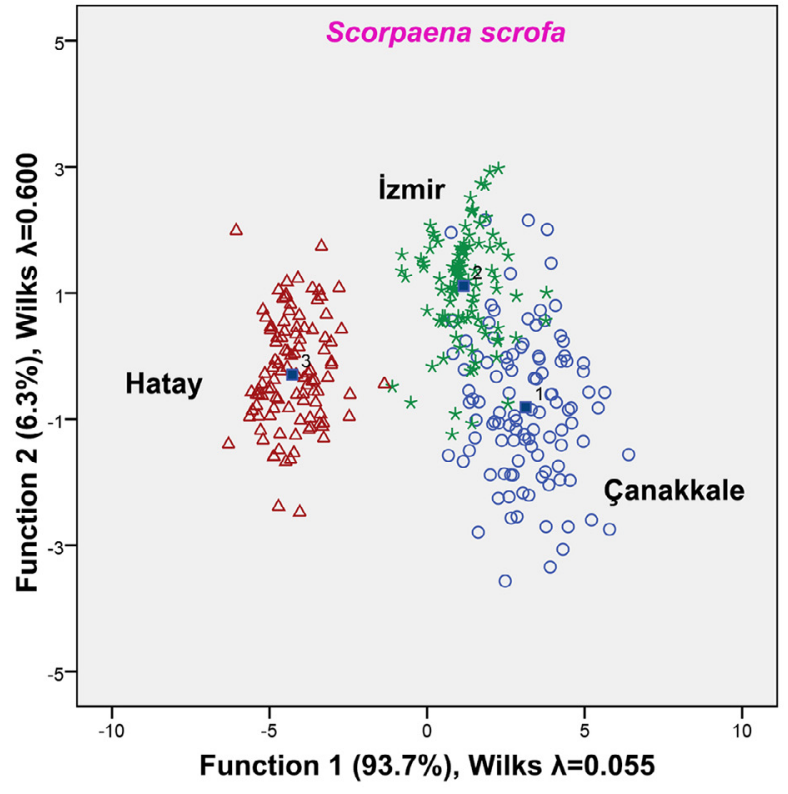

Fig. 8. - Intraspecific discrimination of S. scrofa using morphometric characters.

al height, snout length, maxilla length, eye diameter, pectoral fin base length, anal fin base length, longest anal fin spine length, supraocular tentacle length and ventral fin spine length) were quite important for the interspecific discrimination of five Scorpaena species. These morphometric measurements were selected for the CDA. It was determined that the first four functions were important for the CDA analysis performed for the 
$206 \cdot$ S. Yediel et al.

Table 11. - Descriptive statistics (Mean \pm SD and range) and ANOVA results of morphometric measurements of five Scorpaena species.

\begin{tabular}{|c|c|c|c|c|c|c|c|}
\hline \multirow{2}{*}{$\begin{array}{l}\text { Morphometric } \\
\text { Measurements }\end{array}$} & \multirow{2}{*}{$\begin{array}{l}\text { S. elongata } \\
(\mathrm{n}=332)\end{array}$} & \multirow{2}{*}{$\begin{array}{l}\text { S. maderensis } \\
(\mathrm{n}=326)\end{array}$} & \multirow{2}{*}{$\begin{array}{r}\text { S. notata } \\
(\mathrm{n}=428)\end{array}$} & \multirow{2}{*}{$\begin{array}{l}\text { S. porcus } \\
(\mathrm{n}=459)\end{array}$} & \multirow{2}{*}{$\begin{array}{l}\text { S. scrofa } \\
(\mathrm{n}=320)\end{array}$} & \multicolumn{2}{|c|}{ ANOVA } \\
\hline & & & & & & $\mathrm{F}$ values & $\mathrm{P}$ values \\
\hline TL $(\mathrm{cm})$ & $\begin{array}{c}15.71 \pm 3.57^{\mathrm{b}} \\
(9.2-23.6)\end{array}$ & $\begin{array}{c}12.74 \pm 1.64^{c} \\
(8.7-16.6)\end{array}$ & $\begin{array}{c}15.33 \pm 3.80^{\mathrm{b}} \\
(9.5-22.9)\end{array}$ & $\begin{array}{c}15.90 \pm 4.44^{\mathrm{b}} \\
(8.4-25.1)\end{array}$ & $\begin{array}{c}21.45 \pm 5.05^{\mathrm{a}} \\
(8.9-33.4)\end{array}$ & 214.96 & 0.001 \\
\hline $\mathrm{SL}(\mathrm{cm})$ & $\begin{array}{c}12.60 \pm 1.14^{\mathrm{b}} \\
(7.1-18.9)\end{array}$ & $\begin{array}{c}9.80 \pm 0.23^{\mathrm{d}} \\
(6.7-12.9)\end{array}$ & $\begin{array}{c}11.81 \pm 1.66^{c} \\
(7.2-17.7)\end{array}$ & $\begin{array}{c}12.50 \pm 1.43^{\mathrm{b}} \\
(6.3-19.8)\end{array}$ & $\begin{array}{c}16.68 \pm 0.92^{\mathrm{a}} \\
(7.1-25.6)\end{array}$ & 1347.22 & 0.001 \\
\hline $\mathrm{HL}(\mathrm{cm})$ & $\begin{array}{c}5.21 \pm 0.46^{\mathrm{b}} \\
(2.9-7.9)\end{array}$ & $\begin{array}{c}4.08 \pm 0.17^{d} \\
(2.6-5.6)\end{array}$ & $\begin{array}{c}5.02 \pm 0.84^{\mathrm{c}} \\
(2.7-8.2)\end{array}$ & $\begin{array}{c}4.95 \pm 0.35^{\mathrm{c}} \\
(2.5-8.6)\end{array}$ & $\begin{array}{c}7.29 \pm 0.55^{\mathrm{a}} \\
(3.2-10.7)\end{array}$ & 1612.65 & 0.001 \\
\hline $\mathrm{BL}(\mathrm{mm})$ & $\begin{array}{l}41.16 \pm 4.48^{c} \\
(21.05-68.98)\end{array}$ & $\begin{array}{l}35.07 \pm 1.64^{\mathrm{d}} \\
(22.86-46.20)\end{array}$ & $\begin{array}{l}41.53 \pm 4.59^{c} \\
(25.60-62.95)\end{array}$ & $\begin{array}{l}45.41 \pm 4.78^{\mathrm{b}} \\
(22.30-90.21)\end{array}$ & $\begin{array}{l}54.93 \pm 4.04^{\mathrm{a}} \\
(22.18-82.92)\end{array}$ & 1010.49 & 0.001 \\
\hline CPH (mm) & $\begin{array}{l}11.61 \pm 0.97^{\mathrm{c}} \\
(5.79-19.71)\end{array}$ & $\begin{array}{l}10.05 \pm 0.43^{\mathrm{d}} \\
(6.46-13.64)\end{array}$ & $\begin{array}{l}11.75 \pm 1.84^{\mathrm{c}} \\
(6.72-19.46)\end{array}$ & $\begin{array}{l}12.60 \pm 1.81^{\mathrm{b}} \\
(5.52-22.67)\end{array}$ & $\begin{array}{l}16.99 \pm 1.45^{\mathrm{a}} \\
(6.57-25.29)\end{array}$ & 1041.54 & 0.001 \\
\hline CPL (mm) & $\begin{array}{l}10.48 \pm 1.20^{c} \\
(4.96-20.65)\end{array}$ & $\begin{array}{l}7.27 \pm 0.75^{\mathrm{e}} \\
(4.86-10.45)\end{array}$ & $\begin{array}{l}7.99 \pm 1.57^{d} \\
(4.25-14.71)\end{array}$ & $\begin{array}{l}10.82 \pm 2.79^{b} \\
(4.42-19.32)\end{array}$ & $\begin{array}{l}13.38 \pm 1.19^{\mathrm{a}} \\
(4.47-21.57)\end{array}$ & 663.97 & 0.001 \\
\hline $\mathrm{CL}(\mathrm{cm})$ & $\begin{array}{c}3.14 \pm 0.27^{\mathrm{d}} \\
(1.8-4.8)\end{array}$ & $\begin{array}{c}2.97 \pm 0.13^{\mathrm{e}} \\
(2.0-3.9)\end{array}$ & $\begin{array}{c}3.53 \pm 0.50^{\mathrm{b}} \\
(2.0-5.5)\end{array}$ & $\begin{array}{c}3.45 \pm 0.51^{\mathrm{c}} \\
(1.6-5.4)\end{array}$ & $\begin{array}{c}4.77 \pm 0.47^{\mathrm{a}} \\
(1.7-7.8)\end{array}$ & 916.02 & 0.001 \\
\hline DBL (cm) & $\begin{array}{c}7.60 \pm 0.71^{\mathrm{b}} \\
(4.1-11.8)\end{array}$ & $\begin{array}{c}6.23 \pm 0.23^{\mathrm{d}} \\
(4.2-8.3)\end{array}$ & $\begin{array}{r}7.32 \pm 0.80^{\mathrm{c}} \\
(4.4-11.1)\end{array}$ & $\begin{array}{c}7.73 \pm 0.93^{\mathrm{b}} \\
(3.8-12.6)\end{array}$ & $\begin{array}{c}9.88 \pm 0.53^{\mathrm{a}} \\
(4.4-15.9)\end{array}$ & 1127.34 & 0.001 \\
\hline SDL (mm) & $\begin{array}{l}11.17 \pm 0.80^{c} \\
(7.06-16.23)\end{array}$ & $\begin{array}{l}9.29 \pm 0.64^{\mathrm{e}} \\
(5.74-13.54)\end{array}$ & $\begin{array}{l}10.51 \pm 1.51^{\mathrm{d}} \\
(6.13-17.04)\end{array}$ & $\begin{array}{l}11.58 \pm 1.79^{b} \\
(5.34-19.65)\end{array}$ & $\begin{array}{l}14.68 \pm 1.51^{\mathrm{a}} \\
(6.46-21.33)\end{array}$ & 687.89 & 0.001 \\
\hline LDL (mm) & $\begin{array}{c}21.27 \pm 1.95^{\mathrm{b}} \\
(12.13-32.17)\end{array}$ & $\begin{array}{c}16.31 \pm 0.95^{\mathrm{d}} \\
(11.06-22.10)\end{array}$ & $\begin{array}{l}20.79 \pm 4.12^{\mathrm{b}} \\
(12.22-35.85)\end{array}$ & $\begin{array}{l}19.75 \pm 2.55^{\mathrm{c}} \\
(9.45-30.36)\end{array}$ & $\begin{array}{c}30.29 \pm 3.06^{\mathrm{a}} \\
(12.05-47.17)\end{array}$ & 1105.23 & 0.001 \\
\hline PDL (cm) & $\begin{array}{c}3.93 \pm 0.29^{\mathrm{b}} \\
(2.0-6.6)\end{array}$ & $\begin{array}{c}2.94 \pm 0.14^{\mathrm{d}} \\
(2.0-4.1)\end{array}$ & $\begin{array}{c}3.43 \pm 0.44^{\mathrm{c}} \\
(2.0-5.2)\end{array}$ & $\begin{array}{c}3.85 \pm 0.55^{\mathrm{b}} \\
(1.8-6.5)\end{array}$ & $\begin{array}{c}5.61 \pm 0.39^{\mathrm{a}} \\
(2.1-8.7)\end{array}$ & 2035.03 & 0.001 \\
\hline PVL (cm) & $\begin{array}{c}5.16 \pm 0.48^{b} \\
(2.8-7.9)\end{array}$ & $\begin{array}{c}3.87 \pm 0.13^{\mathrm{d}} \\
(2.7-5.2)\end{array}$ & $\begin{array}{c}4.73 \pm 0.85^{\mathrm{c}} \\
(2.8-8.0)\end{array}$ & $\begin{array}{c}4.82 \pm 0.65^{\mathrm{c}} \\
(2.4-7.9)\end{array}$ & $\begin{array}{c}6.90 \pm 0.45^{\mathrm{a}} \\
(2.7-10.8)\end{array}$ & 1157.40 & 0.001 \\
\hline PAL (cm) & $\begin{array}{r}9.20 \pm 0.84^{\mathrm{b}} \\
(4.8-13.9)\end{array}$ & $\begin{array}{c}6.84 \pm 0.25^{\mathrm{d}} \\
(4.6-9.2)\end{array}$ & $\begin{array}{r}8.33 \pm 1.24^{c} \\
(4.8-13.1)\end{array}$ & $\begin{array}{r}9.12 \pm 1.26^{\mathrm{b}} \\
(4.2-15.7)\end{array}$ & $\begin{array}{c}12.74 \pm 0.86^{\mathrm{a}} \\
(5.1-19.5)\end{array}$ & 1522.82 & 0.001 \\
\hline $\mathrm{POH}(\mathrm{mm})$ & $\begin{array}{l}11.40 \pm 1.03^{b} \\
(6.32-17.12)\end{array}$ & $\begin{array}{l}8.58 \pm 0.41^{\mathrm{cd}} \\
(5.28-11.60)\end{array}$ & $\begin{array}{l}8.69 \pm 1.77^{c} \\
(4.57-15.53)\end{array}$ & $\begin{array}{l}8.37 \pm 1.39^{d} \\
(3.95-14.15)\end{array}$ & $\begin{array}{l}21.66 \pm 1.69^{\mathrm{a}} \\
(9.34-31.91)\end{array}$ & 5742.23 & 0.001 \\
\hline NL (mm) & $\begin{array}{l}12.53 \pm 1.17^{c} \\
(7.03-19.48)\end{array}$ & $\begin{array}{l}10.66 \pm 0.46^{\mathrm{d}} \\
(7.40-14.37)\end{array}$ & $\begin{array}{l}13.51 \pm 2.59^{b} \\
(7.60-24.08)\end{array}$ & $\begin{array}{l}13.46 \pm 2.37^{b} \\
(6.80-21.70)\end{array}$ & $\begin{array}{l}20.10 \pm 2.29^{a} \\
(6.92-32.22)\end{array}$ & 1027.10 & 0.001 \\
\hline ML (mm) & $\begin{array}{l}27.10 \pm 2.41^{b} \\
(15.51-39.46)\end{array}$ & $\begin{array}{c}20.64 \pm 0.71^{\mathrm{e}} \\
(13.90-27.41)\end{array}$ & $\begin{array}{l}25.31 \pm 4.21^{d} \\
(15.62-41.76)\end{array}$ & $\begin{array}{c}26.47 \pm 3.40^{c} \\
(13.36-43.47)\end{array}$ & $\begin{array}{c}37.04 \pm 2.58^{a} \\
(14.94-54.26)\end{array}$ & 1270.19 & 0.001 \\
\hline $\mathrm{ED}(\mathrm{mm})$ & $\begin{array}{l}15.48 \pm 3.04^{b} \\
(6.15-24.78)\end{array}$ & $\begin{array}{c}9.23 \pm 0.39^{\mathrm{e}} \\
(7.02-11.60)\end{array}$ & $\begin{array}{l}10.98 \pm 2.04^{\mathrm{d}} \\
(6.55-18.87)\end{array}$ & $\begin{array}{l}11.37 \pm 2.68^{c} \\
(4.43-20.58)\end{array}$ & $\begin{array}{c}17.27 \pm 0.84^{\mathrm{a}} \\
(10.95-22.16)\end{array}$ & 843.05 & 0.001 \\
\hline IOD (mm) & $\begin{array}{l}6.05 \pm 0.60^{c} \\
(3.25-8.84)\end{array}$ & $\begin{array}{l}5.56 \pm 026^{\mathrm{d}} \\
(3.54-7.84)\end{array}$ & $\begin{array}{l}6.81 \pm 1.27^{\mathrm{b}} \\
(4.12-11.89)\end{array}$ & $\begin{array}{l}6.94 \pm 1.47^{\mathrm{b}} \\
(3.45-12.95)\end{array}$ & $\begin{array}{c}9.65 \pm 1.25^{\mathrm{a}} \\
(3.75-14.99)\end{array}$ & 646.45 & 0.001 \\
\hline PBL (mm) & $\begin{array}{l}16.01 \pm 1.33^{d} \\
(9.36-23.47)\end{array}$ & $\begin{array}{l}13.78 \pm 0.61^{\mathrm{e}} \\
(8.65-18.83)\end{array}$ & $\begin{array}{l}16.61 \pm 2.60^{c} \\
(9.84-27.44)\end{array}$ & $\begin{array}{l}18.58 \pm 2.54^{\mathrm{b}} \\
(9.12-30.65)\end{array}$ & $\begin{array}{l}23.46 \pm 2.69^{\mathrm{a}} \\
(9.69-35.05)\end{array}$ & 973.11 & 0.001 \\
\hline PPL (cm) & $\begin{array}{c}5.11 \pm 0.50^{\mathrm{b}} \\
(2.6-8.3)\end{array}$ & $\begin{array}{c}3.68 \pm 0.14^{\mathrm{d}} \\
(2.6-4.8)\end{array}$ & $\begin{array}{c}4.39 \pm 0.67^{\mathrm{c}} \\
(2.7-6.9)\end{array}$ & $\begin{array}{c}4.48 \pm 0.60^{\mathrm{c}} \\
(2.3-7.8)\end{array}$ & $\begin{array}{r}6.49 \pm 0.32^{\mathrm{a}} \\
(2.5-11.2)\end{array}$ & 1419.14 & 0.001 \\
\hline VBL (mm) & $\begin{array}{l}4.90 \pm 1.11^{\mathrm{c}} \\
(1.55-9.56)\end{array}$ & $\begin{array}{l}4.46 \pm 0.23^{\mathrm{d}} \\
(3.10-5.81)\end{array}$ & $\begin{array}{l}5.15 \pm 0.66^{\mathrm{b}} \\
(3.00-7.80)\end{array}$ & $\begin{array}{l}5.24 \pm 0.46^{\mathrm{b}} \\
(1.68-8.95)\end{array}$ & $\begin{array}{l}7.04 \pm 0.85^{\mathrm{a}} \\
(1.95-11.58)\end{array}$ & 619.24 & 0.001 \\
\hline VSL (mm) & $\begin{array}{c}19.01 \pm 1.24^{b} \\
(12.39-25.88)\end{array}$ & $\begin{array}{c}14.64 \pm 0.54^{\mathrm{e}} \\
(10.70-18.03)\end{array}$ & $\begin{array}{c}17.18 \pm 2.94^{\mathrm{d}} \\
(10.40-27.26)\end{array}$ & $\begin{array}{l}18.34 \pm 2.66^{c} \\
(9.68-28.99)\end{array}$ & $\begin{array}{l}24.57 \pm 1.92^{\mathrm{a}} \\
(12.68-33.59)\end{array}$ & 931.15 & 0.001 \\
\hline ABL (mm) & $\begin{array}{c}18.03 \pm 1.68^{\mathrm{d}} \\
(11.19-26.05)\end{array}$ & $\begin{array}{c}16.84 \pm 0.78^{\mathrm{e}} \\
(12.05-21.25)\end{array}$ & $\begin{array}{c}18.97 \pm 1.72^{\mathrm{c}} \\
(12.48-25.95)\end{array}$ & $\begin{array}{l}19.68 \pm 2.42^{\mathrm{b}} \\
(9.58-29.54)\end{array}$ & $\begin{array}{l}23.27 \pm 1.69^{\mathrm{a}} \\
(11.71-37.16)\end{array}$ & 593.89 & 0.001 \\
\hline SAL (mm) & $\begin{array}{c}8.12 \pm 0.67^{\mathrm{e}} \\
(5.41-11.36)\end{array}$ & $\begin{array}{l}9.46 \pm 0.38^{\mathrm{d}} \\
(6.72-11.97)\end{array}$ & $\begin{array}{l}10.46 \pm 1.04^{c} \\
(6.43-15.43)\end{array}$ & $\begin{array}{l}10.99 \pm 1.56^{\mathrm{b}} \\
(5.63-17.66)\end{array}$ & $\begin{array}{l}12.13 \pm 1.23^{\mathrm{a}} \\
(5.28-18.69)\end{array}$ & 644.06 & 0.001 \\
\hline LAL (mm) & $\begin{array}{c}19.09 \pm 1.58^{c} \\
(11.91-27.77)\end{array}$ & $\begin{array}{c}17.41 \pm 0.65^{\mathrm{d}} \\
(13.11-21.63)\end{array}$ & $\begin{array}{c}19.80 \pm 2.10^{\mathrm{b}} \\
(12.84-27.71)\end{array}$ & $\begin{array}{c}19.26 \pm 2.17^{\mathrm{c}} \\
(10.00-27.95)\end{array}$ & $\begin{array}{c}23.63 \pm 1.38^{\mathrm{a}} \\
(11.70-34.56)\end{array}$ & 568.49 & 0.001 \\
\hline STL (mm) & $\begin{array}{l}2.19 \pm 0.49^{\circ} \\
(0.74-3.28)\end{array}$ & $\begin{array}{l}4.32 \pm 0.33^{\mathrm{b}} \\
(3.21-5.90)\end{array}$ & $\begin{array}{l}4.17 \pm 1.57^{\mathrm{b}} \\
(1.83-9.29)\end{array}$ & $\begin{array}{l}11.34 \pm 2.63^{\mathrm{a}} \\
(4.43-20.58)\end{array}$ & $\begin{array}{l}1.70 \pm 0.24^{\mathrm{d}} \\
(1.10-2.22)\end{array}$ & 2656.61 & 0.001 \\
\hline
\end{tabular}

Table 12. - Jackknife classification matrix of the discriminant canonical analysis applied to the five Scorpaena species from the coastline of Turkey's four seas.

\begin{tabular}{|c|c|c|c|c|c|}
\hline \multirow[b]{2}{*}{ Species } & \multicolumn{5}{|c|}{ Predicted Group Membership } \\
\hline & S. elongata & S. maderensis & S. notata & S. porcus & S. scrofa \\
\hline S. elongata & $98.5(327)$ & $1.5(5)$ & - & - & - \\
\hline S. maderensis & $1.8(6)$ & $98.2(320)$ & - & - & \\
\hline S. notata & - & - & $96.7(414)$ & $3.3(14)$ & \\
\hline S. porcus & - & - & $5.2(24)$ & $94.8(435)$ & \\
\hline S. scrofa & & & & & $100.0(320)$ \\
\hline
\end{tabular}

The correct classifications percentages and numbers are in bold; the number of individuals is given in parentheses. 


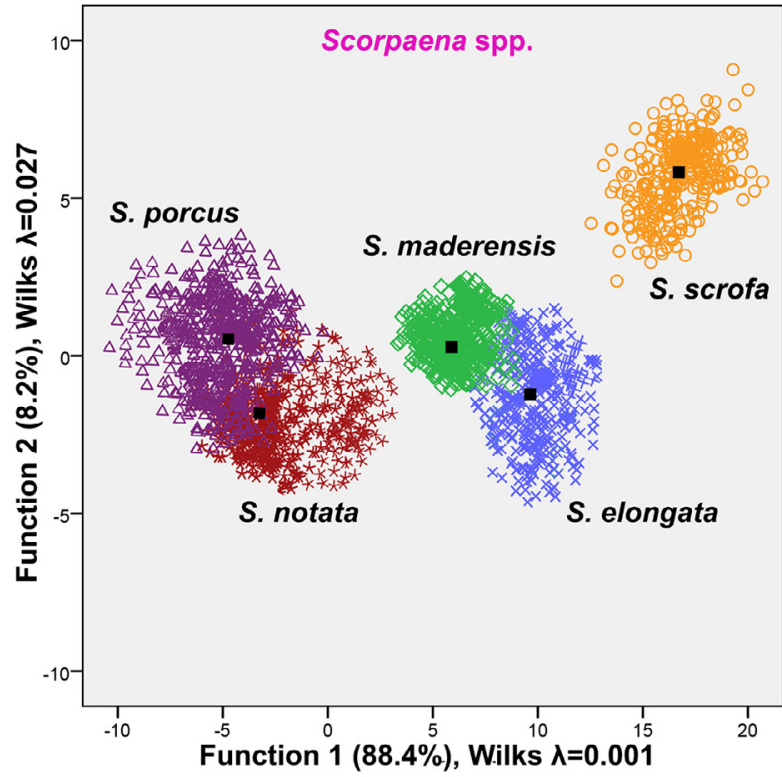

Fig. 9. - Interspecific discrimination of the five Scorpaena species using morphometric characters

five Scorpaena species (F1[88.4\%], $\lambda=0.001, \mathrm{P}<0.001$; $\mathrm{F} 2[8.2 \%], \quad \lambda=0.027, \quad \mathrm{P}<0.001 ; \mathrm{F} 3[2.0 \%], \quad \lambda=0.194$, $\mathrm{P}<0.001 ; \mathrm{F} 4[1.4 \%], \lambda=0.493, \mathrm{P}<0.001$ ) (Fig. 9). It is determined from the CDA results that these 13 characters taken from the fish samples were quite effective for discriminating five Scorpaena species from each other and that they achieved $97.4 \%$ success in interspecific discrimination of these Scorpaena species (Table 12).

Hierarchical cluster analyses of meristic data clustered Scorpaena species. Three main branches were produced by UPGMA. In the first branch, $S$. scrofa was seen to be morphometrically most divergent from the other species. In the second branch, $S$. notata and $S$. porcus were the closest taxa forming the sister group to $S$. elongata. The neighbouring branch made up of $S$. maderensis was seen to be morphometrically most divergent from the other species and was branched as a third group. The third group, the neighbouring branch, included only $S$. maderensis (Fig. 4).

\section{DISCUSSION}

The genus Scorpaena is distributed throughout temperate and tropical seas of the world (Hureau and Litvinenko 1986, Gomon et al. 1994, Froese and Pauly 2020). It is known that Scorpaena species are difficult to identify at the species level using visual observation alone due to colouration similarities and overlapping morphological features in different habitats (Hureau and Livtinenko 1986, Golani et al. 2006, Akalın et al. 2011). Morphometric characters of the fish species are a strong means to measure and distinguish species and stock relations (Turan et al. 2005, Cadrin et al. 2014). In this study, the intra- and interspecific discriminations of five Scorpaena species inhabiting the Aegean, Black, Mediterranean and Marmara seas were successfully performed using CDA based on morphometric characters. It was determined that data obtained from
Scorpaena species showed some differences among the species but were generally compatible with the data of Froese and Pauly (2020). The most significant measurements of five Scorpaena species taken into account for discrimination through the traditional analysis were body height, caudal peduncle height, caudal peduncle length, longest dorsal fin spine length, preorbital height, snout length, maxilla length, eye diameter, pectoral fin base length, anal fin base length, longest anal fin spine length, supraocular tentacle length and ventral fin spine length. As a result of CDA analysis, it was determined that morphometric characters are also effective for intraspecific discrimination of five Scorpaena species. For example, the highest intraspecific discrimination was determined for $S$. notata populations $(96.7$ $\%)$, followed by S. porcus (96.5\%), S. elongata (94.6 $\%)$, S. scrofa $(92.2 \%)$ and S. maderensis $(90.5 \%)$. The intraspecific morphological variations of five Scorpaena species may be due to variation in body shape but not to the total length effect because it was normalized successfully using the Elliott et al. (1995) method. Cadrin (2000) indicated that is difficult to explain the causes of morphological differences between fish populations. However, it is assumed that these differences may be related to genetic factors or may also be related to environmental factors such as feeding, habitat, $\mathrm{pH}$, turbidity and temperature (Wimberger 1992).

Pothin et al. (2006) indicated that the Wilks' lambda $(\lambda)$ value varies between 0 and 1 . The discriminating power of CDA is best when Wilks' lambda $(\lambda)$ is close to 0 . In the current study, the Wilks' lambda $(\lambda)$ values for intraspecific discriminations were 0.008 for $S$. elongata, 0.110 for $S$. maderensis, 0.003 for $S$. notata, 0.002 for $S$. porcus and 0.055 for $S$. scrofa. The Wilks' lambda $(\lambda)$ value was determined as 0.001 for interspecific discrimination of the five Scorpaena species. CDA results show that morphological measurements of the five Scorpaena species produce good discrimination within each species and among the species. These lambda values also support the high accuracy of CDA for morphometric measurements in the present study. Using the morphometric measurements of Scorpaena species, the actual separation rate in CDA was determined to be high (Table 12). Body morphometric traits were reported to provide a moderate level of discrimination in many species and genera from marine and freshwater habitats such as Trachurus mediterraneus in the Aegean, Black, and Mediterranean seas (Turan 2004), Eugerres spp. in the eastern Pacific (González-Acosta et al. 2005), Megalaspis cordyla from the Indian coast (Sajina et al. 2011), the genus Labeo in Assam, India (Choudhury and Dutta 2012), Channa punctatus from Indian rivers (Khan et al. 2013), the genus Nemipterus in Malaysia and its surrounding seas (Imtiaz and Naim 2018), Ompok pabo from Bangladeshi freshwaters (Mahfuj et al. 2019b) and mullet species in Aceh, Indonesia (Yulianto et al. 2020).

La Mesa (2005) revised the description of $S$. maderensis sampled from the southeastern coasts of Sicily using the metric and meristic characteristics and reported that most of the morphometric characters of $S$. maderensis evaluated in this study overlap with the 
$S$. porcus data, causing some problems in species distinction. The same author reported that supraocular tentacle length and anal fin spine length were the most effective characters for distinguishing $S$. maderensis and $S$. porcus species. Similarly, in the present study, it was determined that the supraocular tentacle length, shortest anal fin spine length and longest anal fin spine length were the most effective characters for distinguishing between $S$. maderensis and $S$. porcus species. Turan et al. (2009) compared S. elongata, S. maderensis, $S$. notata, $S$. porcus, and $S$. scrofa from Iskenderun Bay (Mediterranean Sea) based on the number of spines and soft rays on anal, ventral and dorsal fins, the number of soft rays on the pectoral and caudal fins, the number of scales on the lateral line, and the number of gill spines and vertebrae. They concluded that caudal fin rays, pectoral fin rays, vertebrae numbers and lateral scale numbers are important for species differentiation.

Ferri et al. (2010) evaluated 18 morphometric characteristics of $S$. porcus sampled from the eastern Adriatic Sea. They reported that these characters did not differ statistically between male and female individuals $(P>0.05)$. Similarly, in the present study, 26 morphometric characters were evaluated for $S$. porcus from the Aegean, Black, Mediterranean seas and Marmara seas, and it was determined that metric characters showed no statistical difference between female and male individuals ( $\mathrm{P}>0.05)$. Thus, this study contributes to the literature by supporting the data of previous studies over new samples obtained from different stations in different habitats. Akalın et al. (2011) compared 19 metric and 7 meristic characteristics in $S$. porcus and $S$. notata sampled from the Aegean Sea. Although they stated that the black spot on the dorsal fin and supraocular tentacle are effective characters for differentiating these two species, there were problems in distinguishing juvenile individuals. Therefore, they stated that a detailed morphometric comparison was needed for Scorpaena species. They found statistical differences between the two species in supraocular tentacle length, upper jaw length, pectoral fin length, caudal peduncle height, eye diameter, longest dorsal fin spine length, pelvic fin spine length, shortest anal fin spine length and longest anal fin spine length. Our study also revealed that morphometric characters may differ statistically among fish species (Table 11). Manilo and Peskov (2016) evaluated 20 morphometric characteristics of $S$. porcus sampled from the south coast of Crimea and the eastern part of the Adriatic Sea. They compared the male and female individuals in both regions separately. As a result of this comparison, they reported that the 13 morphometric characteristics were statistically different between these two regions. Similar results were obtained in the present study, and the PCA indicated that 13 morphometric measurements were important for the intraspecific discrimination of five $S$. porcus from four seas. We also achieved a success rate of $96.5 \%$ in the intraspecific separation of $S$. porcus sampled from different seas using these morphometric data (Table 8). As explained above in the literature review, although there are some intra- and interspecific morphometric-based studies for the genus Scorpaena, no studies based on morphometric measurements of these five Scorpaena species had been carried out in such wide geographic areas. Therefore, this is the first study based on morphometric data to perform intra- and interspecific discrimination of the five Scorpaena species sampled from eight stations in four seas.

In many studies, the characters are considered one of the simplest, most cost-effective and most commonly used tools to distinguish between fish populations (Khan et al. 2013, Siddik et al. 2015), to determine the structure of fish assemblages (Cheng et al. 2005) and to identify fish stocks (Cadrin et al. 2014, Siddik et al. 2016). However, in some cases these morphometric characters may not be suitable for identifying or discriminating every fish species and population. Therefore, the determination of these characters is important for fish biology and fisheries management.

\section{ACKNOWLEDGEMENTS}

This study is part of the PhD thesis of the first author. The study was supported by Ordu University Scientific Research Projects Commission (BAP; B-1917). The authors thank the Commission and we also thank the anonymous reviewers for comments that greatly improved the manuscript.

\section{REFERENCES}

Akalın S., İlhan D., Ünlüoğlu A., et al. 2011. Length-weight relationship and metric-meristic characteristics of two scorpion fishes (Scorpaena notata and Scorpaena porcus) in İzmir Bay. J. Fish. Sci. 5: 291-299. https://doi.org/10.3153/jfscom.2011033

Arculeo M., Lo Brutto S. 2014. New contribution to the systematic status of various Mediterranean Scorpionfish, as inferred from a mitochondrial DNA sequence. Rev. Biol. Mar. Oceanogr. 49: 367-371. https://doi.org/10.4067/S0718-19572014000200015

Baldwin R.E., Banks M.A., Jacobson K.C. 2012. Integrating fish and parasite data as a holistic solution for identifying the elusive stock structure of Pacific sardines (Sardinops sagax). Rev. Fish. Biol. Fisher. 22: 137-156. https://doi.org/10.1007/s11160-011-9227-5

Batubara A.S., Muchlisin Z.A., Efizon D., et al. 2018. Morphometric variations of the genus Barbonymus (Pisces, Cyprinidae) harvested from Aceh Waters, Indonesia. Fish. Aquatic. Sci. 26: 231-237. https://doi.org/10.2478/aopf-2018-0026

Cadrin S.X. 2000. Advances in morphometric identification of fishery stocks. Rev. Fish. Biol. Fisher. 10: 91-112. https://doi.org/10.1023/A:1008939104413

Cadrin S.X., Kerr L.A., Mariani S. 2014. Stock identification methods: an overview. In: Cadrin S.X., Kerr L.A., Mariani S. (eds), Stock Identification Methods. San Diego: Elsevier, pp. $1-5$. https://doi.org/10.1016/B978-0-12-397003-9.00001-1

Caputo V., Sorice M., Vitturi R., et al. 1998. Cytogenetic studies in some species of Scorpaeniformes (Teleostei: Percomorpha). Chromosome Res. 6: 255-262. https://doi.org/10.1023/A:1009210605487

Cheng Q., Lu D., Ma L. 2005. Morphological differences between close populations discernible by multivariate analysis: a case study of genus Coilia (Teleostei: Clupeiforms). Aquat. Living Resour. 18: 187-192. https://doi.org/10.1051/alr:2005020

Choudhury S., Dutta K. 2012. Interrelationships of five species of the genus Labeo by morphometric analysis. IOSR J. Pharm. Biol. Sci. 2: 35-39. https://doi.org/10.9790/3008-0263337 
Choudhury S., Saikia P., Sougrakpam N., et al. 2011. Assessment of morphometric variation and establishing taxonomic relationship among six species under Puntius genus. Int. J. Environ. Res. 1: 233-237.

Elliott N.G., Haskard K., Koslow J.A. 1995. Morphometric analysis of orange roughy (Hoplostethus atlanticus) off the continental slope of southern Australia. J. Fish Biol. 46: 202-220. https://doi.org/10.1111/j.1095-8649.1995.tb05962.x

Ferri J., Petrić M., Matić-Skoko S. 2010. Biometry analysis of the black scorpionfish, Scorpaena porcus (Linnaeus, 1758) from the eastern Adriatic Sea. Acta Adriat. 51: 45-53.

Fischer W., Schneider M., Bauchot M.L. 1987. Fiches FAO d'identification des espèces pour les besoins de la pêche. Méditerranée et mer Noire. Zone de Pêche 37. Vol. II. Vertébrés. FAO, Rome, $1070 \mathrm{pp}$. http://www.fao.org/3/x0170f/x0170f00.htm

Froese R., Pauly D. 2020. FishBase, World Wide Web electronic publication. Accessed: 12.12.2020. http://www.fishbase.org/

Golani D., Ozturk B., Bassusta N. 2006. Fishes of the eastern Mediterranean. Turkish Marine Research Foundation, Istanbul, $260 \mathrm{pp}$

Gomon M.F., Glover J.C.M., Kuiter R.H. 1994. The fishes of Australia's south coast. State Print, Adelaide, 992 pp.

González-Acosta A.F., De La Cruz-Agüero J., Castro-Aguirre J.L. 2005. A review of eastern Pacific species of the genus Eugerres (Perciformes: Gerreidae). Bull. Mar. Sci. 76: 661673

Hureau J.C., Litvinenko N.I. 1986. Scorpaenidae. In: Whitehead P.J.P., Bauchot M.L., et al. (eds), Fishes of the North-eastern Atlantic and the Mediterranean, Paris: Unesco, pp. 12111229.

Ibáñez A.L., Jawad L.A. 2018. Morphometric variation of fish scales among some species of rattail fish from New Zealand waters. J. Mar. Biol. Assoc. U.K. 98: 1991-1998. https://doi.org/10.1017/S0025315418000024

Imtiaz A., Naim D.M.D. 2018. Geometric morphometrics species discrimination within the genus Nemipterus from Malaysia and its surrounding seas. Biodiversitas 19: 2316-2322. https://doi.org/10.13057/biodiv/d190640

Jayasankar P., Thomas P.C., Paulton M.P., et al. 2004. Morphometric and genetic analyzes of Indian mackerel (Rastrelliger kanagurta) from peninsular India. Asian Fish. Sci. 17: 201215.

Keskin Ç., Eryılmaz L. 2009. The presence of Scorpaena loppei (Osteichthyes: Scorpaenidae), in the Turkish seas. Mar. Biodivers. Rec. 2: 1-2. https://doi.org/10.1017/S175526720800034

Khan M.A., Miyan K., Khan S. 2013. Morphometric variation of snakehead fish, Channa punctatus, populations from three Indian rivers. J. Appl. Ichthyol. 29: 637-642. https://doi.org/10.1111/j.1439-0426.2012.02058.x

La Mesa G. 2005. A revised description of Scorpaena maderensis (Scorpaenidae) by means of meristic and morphometric analysis. J. Mar. Biol. Assoc. U.K. 85: 1263-1270. https://doi.org/10.1017/S0025315405012415

Mahfuj M.S., Khatun A., Boidya P., et al. 2019a. Meristic and morphometric variations of barred spiny eel, Macrognathus pancalus populations from Bangladeshi freshwaters: an insight into landmark-based truss network system. Ribarstvo 77: 7-18. https://doi.org/10.2478/cjf-2019-0002

Mahfuj M.S., Hossain M.F., Jinia S.S., et al. 2019b. Meristic and morphometric variations of critically endangered butter catfish, Ompok pabo inhabiting three natural sources. Int. J. Biosci. 14: 518-527.

Manilo L.G., Peskov V.N. 2016. Comparative morphometric analysis of the small-scaled scorpionfish, Scorpaena porcus (Scorpaenidae, Scorpaeniformes), from the southern coast of the Crimea and eastern part of the Adriatic Sea. Vestn. Zool. 50: 533-538. https://doi.org/10.1515/vzoo-2016-0060

Morato T., Afonso P., Lourinho P., et al. 2001. Length-weight relationships for 21 coastal fish species of the Azores, North-Eastern Atlantic. Fish. Res. 50: 297-302. https://doi.org/10.1016/S0165-7836 (00)00215-0

Nelson J.S., Grande T.C., Wilson M.V.H. 2016. Fishes of the world. John Wiley \& Sons, Inc, New Jersey, 707 pp. https://doi.org/10.1002/9781119174844
Pothin K., Gonzales-Salas C., Chabanet P., et al. 2006. Distinction between Mulloidichthys flavolineatus juveniles from Reunion Island and Mauritius Island (south-west Indian Ocean) based on otolith morphometrics. J. Fish Biol. 69: $38-53$ https://doi.org/10.1111/j.1095-8649.2006.01047.x

Rawat S., Benakappa S., Jitendra Kumar A.S., et al. 2017. Identification of fish stocks based on truss morphometric: A review. J. Fish. Life Sci. 2: 9-14.

Sajina A.M., Chakraborty S.K., Jaiswar A.K., et al. 2011. Stock structure analysis of Megalaspis cordyla (Linnaeus, 1758 ) along the Indian coast based on truss network analysis. Fish. Res. 108: 100-105. https://doi.org/10.1016/i.fishres.2010.12.006

Siddik M.A.B., Hanif M.A., Chaklader M.R., et al. 2015. Fishery biology of gangetic whiting Sillaginopsis panijus (Hamilton, 1822) endemic to Ganges delta, Bangladesh. Egypt. J. Aquat. Res. 41: 307-313. https://doi.org/10.1016/j.ejar.2015.11.001

Siddik M.A.B., Chaklader M.R., Hanif M.A., et al. 2016. Stock identification of critically endangered olive barb, Puntius sarana (Hamilton, 1822) with emphasis on management implications. J. Aquac. Res. Dev. 7: 1-6. https://doi.org/10.4172/2155-9546.1000411

Turan C. 2004. Stock identification of Mediterranean horse mackerel (Trachurus mediterraneus) using morphometric and meristic characters. ICES J. Mar. Sci. 61: 774-781. https://doi.org/10.1016/j.icesjms. 2004.05.001

Turan C., Yalcin S., Turan F., et al. 2005. Morphometric comparisons of African catfish, Clarias gariepinus, populations in Turkey. Folia Zool. 54: 165-172.

Turan C., Oral M., Öztürk B., et al. 2006. Morphometric and meristic variation between stocks of Bluefish (Pomatomus saltatrix) in the Black, Marmara, Aegean and northeastern Mediterranean Seas. Fish. Res. 79: 139-147. https://doi.org/10.1016/j.fishres.2006.01.015

Turan C., Gündüz I., Gurlek M., et al. 2009. Systematics of Scorpaeniformes species in the Mediterranean Sea inferred from mitochondrial 16s rDNA sequence and morphological data. Folia Biol. 57: 219-226. https://doi.org/10.3409/fb57 1-2.219-226

Ujjainia N.C., Kohli M.P.S. 2011. Landmark-based morphometric analysis for selected species of Indian major carp (Catla catla, Ham. 1822). Int. J. Food Agric. Vet. Sci. 1: 64-74.

Wimberger P.H. 1992. Plasticity of fish body shape. The effects of diet, development, family and age in two species of Geophagus (Pisces: Cichlidae). Biol. J. Linn. Soc. 45: 197-218. https://doi.org/10.1111/j.1095-8312.1992.tb00640.x

Yulianto D., Indra I., Batubara A.S., et al. 2020. Morphometrics and genetics variations of mullets (Pisces: Mugillidae) from Aceh waters, Indonesia. Biodiversitas 21: 3422-3430. https://doi.org/10.13057/biodiv/d210802

\section{SUPPLEMENTARY MATERIAL}

The following supplementary material is available through the online version of this article and at the following link: http://scimar.icm.csic.es/scimar/supplm/ sm05185esm.pdf

Table S1. - Ranges of selected morphometric variables of S. elongata populations.

Table S2. - Ranges of selected morphometric variables of S. maderensis populations.

Table S3. - Ranges of selected morphometric variables of S. notata populations.

Table S4. - Ranges of selected morphometric variables of S. porcus populations.

Table S5. - Ranges of selected morphometric variables of S. scrofa populations. 
Scientia Marina 85(3)

September 2021, S1-S3, Barcelona (Spain)

ISSN-L: 0214-8358

\section{Intra- and interspecific discrimination of Scorpaena} species from the Aegean, Black, Mediterranean and Marmara seas

Serdar Yedier, Derya Bostanci

Supplementary material 
Table S1. - Ranges of selected morphometric variables of S. elongata populations.

\begin{tabular}{lccc}
\hline $\begin{array}{l}\text { Morphometric } \\
\text { Variables }\end{array}$ & Antalya & Hatay & İzmir \\
\hline $\mathrm{BL} / \mathrm{SL}$ & $0.293-0.365$ & $0.296-0.325$ & $0.324-0.346$ \\
$\mathrm{LDL} / \mathrm{SL}$ & $0.170-0.194$ & $0.164-0.171$ & $0.162-0.168$ \\
$\mathrm{PAL} / \mathrm{SL}$ & $0.667-0.735$ & $0.732-0.746$ & $0.730-0.733$ \\
$\mathrm{POH} / \mathrm{SL}$ & $0.088-0.091$ & $0.089-0.092$ & $0.092-0.096$ \\
$\mathrm{NL} / \mathrm{SL}$ & $0.098-0.103$ & $0.098-0.099$ & $0.097-0.101$ \\
$\mathrm{ML} / \mathrm{SL}$ & $0.209-0.215$ & $0.213-0.228$ & $0.211-0.230$ \\
$\mathrm{ED} / \mathrm{SL}$ & $0.131-0.161$ & $0.136-0.175$ & $0.081-0.083$ \\
$\mathrm{PBL} / \mathrm{SL}$ & $0.124-0.130$ & $0.122-0.133$ & $0.121-0.147$ \\
$\mathrm{VBL} / \mathrm{SL}$ & $0.022-0.044$ & $0.028-0.046$ & $0.023-0.051$ \\
$\mathrm{VSL} / \mathrm{SL}$ & $0.137-0.172$ & $0.146-0.180$ & $0.136-0.167$ \\
$\mathrm{ABL} / \mathrm{SL}$ & $0.138-0.155$ & $0.136-0.155$ & $0.137-0.156$ \\
$\mathrm{LAL} / \mathrm{SL}$ & $0.147-0.167$ & $0.136-0.168$ & $0.145-0.180$ \\
\hline
\end{tabular}

Standard length (SL), body height (BL), longest dorsal fin spine length (LDL), preanal length (PAL), preorbital height (POH), snout length $(\mathrm{NL})$, maxilla length (ML), eye diameter (ED), pectoral fin base length (PBL), ventral fin base length (VBL), ventral fin spine length (VSL), anal fin base length (ABL) and longest anal fin spine length (LAL).

Table S2. - Ranges of selected morphometric variables of $S$. maderensis populations.

\begin{tabular}{lccc}
\hline $\begin{array}{l}\text { Morphometric } \\
\text { Variables }\end{array}$ & Antalya & Balıkesir & İzmir \\
\hline $\mathrm{BL} / \mathrm{SL}$ & $0.362-0.370$ & $0.361-0.375$ & $0.336-0.347$ \\
$\mathrm{LDL} / \mathrm{SL}$ & $0.165-0.169$ & $0.172-0.181$ & $0.155-0.165$ \\
$\mathrm{PAL} / \mathrm{SL}$ & $0.697-0.724$ & $0.701-0.703$ & $0.667-0.676$ \\
$\mathrm{POH} / \mathrm{SL}$ & $0.088-0.091$ & $0.088-0.091$ & $0.078-0.088$ \\
$\mathrm{ML} / \mathrm{SL}$ & $0.207-0.210$ & $0.214-0.227$ & $0.204-0.210$ \\
$\mathrm{CPH} / \mathrm{SL}$ & $0.107-0.110$ & $0.096-0.103$ & 0.10 \\
$\mathrm{CPL} / \mathrm{SL}$ & $0.082-0.089$ & $0.062-0.073$ & $0.070-0.073$ \\
$\mathrm{PBL} / \mathrm{SL}$ & $0.142-0.146$ & $0.140-0.147$ & $0.128-0.138$ \\
$\mathrm{ABL} / \mathrm{SL}$ & $0.164-0.180$ & $0.166-0.189$ & $0.165-0.180$ \\
$\mathrm{LAL} / \mathrm{SL}$ & $0.169-0.196$ & $0.169-0.202$ & $0.158-0.196$ \\
\hline
\end{tabular}

Standard length (SL), body height (BL), caudal peduncle height (CPH), caudal peduncle length (CPL), longest dorsal fin spine length (LDL), preanal length (PAL), preorbital height (POH), maxilla length (ML), pectoral fin base length (PBL), anal fin base length (ABL) and longest anal fin spine length (LAL).

Table S3. - Ranges of selected morphometric variables of S. notata populations.

\begin{tabular}{lcccc}
\hline $\begin{array}{l}\text { Morphometric } \\
\text { Variables }\end{array}$ & Hatay & İzmir & $\begin{array}{c}\text { Marmara } \\
\text { Ereğlisi }\end{array}$ & Şile \\
\hline BL/SL & $0.337-0.362$ & $0.356-0.362$ & $0.357-0.361$ & $0.349-0.356$ \\
LDL/SL & $0.175-0.193$ & $0.159-0.203$ & $0.160-0.174$ & $0.150-0.170$ \\
$\mathrm{POH} / \mathrm{SL}$ & $0.069-0.078$ & $0.054-0.088$ & $0.063-0.071$ & $0.070-0.073$ \\
$\mathrm{NL} / \mathrm{SL}$ & $0.109-0.130$ & $0.108-0.136$ & $0.109-0.116$ & $0.106-0.117$ \\
$\mathrm{ML} / \mathrm{SL}$ & $0.221-0.236$ & $0.204-0.236$ & $0.201-0.225$ & $0.208-0.217$ \\
$\mathrm{ED} / \mathrm{SL}$ & $0.089-0.104$ & $0.089-0.107$ & $0.080-0.098$ & $0.082-0.094$ \\
$\mathrm{PBL} / \mathrm{SL}$ & $0.136-0.155$ & $0.140-0.154$ & $0.134-0.137$ & $0.137-0.141$ \\
$\mathrm{CPH} / \mathrm{SL}$ & $0.102-0.110$ & $0.096-0.109$ & $0.096-0.099$ & $0.093-0.101$ \\
$\mathrm{CPL} / \mathrm{SL}$ & $0.064-0.081$ & $0.059-0.083$ & $0.062-0.069$ & $0.059-0.071$ \\
$\mathrm{ABL} / \mathrm{SL}$ & $0.145-0.183$ & $0.147-0.176$ & $0.147-0.179$ & $0.152-0.173$ \\
$\mathrm{LAL} / \mathrm{SL}$ & $0.157-0.197$ & $0.154-0.182$ & $0.145-0.178$ & $0.155-0.192$ \\
$\mathrm{VSL} / \mathrm{SL}$ & $0.147-0.154$ & $0.154-0.156$ & $0.128-0.144$ & $0.127-0.150$ \\
$\mathrm{STL} / \mathrm{SL}$ & $0.025-0.032$ & $0.037-0.052$ & $0.039-0.042$ & $0.025-0.027$ \\
\hline
\end{tabular}

Standard length (SL), body height (BL), caudal peduncle height $(\mathrm{CPH})$, caudal peduncle length (CPL), longest dorsal fin spine length $(\mathrm{LDL})$, preorbital height $(\mathrm{POH})$, snout length (NL), maxilla length (ML), eye diameter (ED), pectoral fin base length (PBL), ventral fin spine length (VSL), anal fin base length (ABL), longest anal fin spine length (LAL) and supraocular tentacle length (STL). 
Table S4. - Ranges of selected morphometric variables of $S$. porcus populations.

\begin{tabular}{lcccc}
\hline $\begin{array}{l}\text { Morphometric } \\
\text { Variables }\end{array}$ & Hatay & İzmir & $\begin{array}{c}\text { Marmara } \\
\text { Ereğlisi }\end{array}$ & Ordu \\
\hline BL/SL & $0.352-0.394$ & $0.357-0.456$ & $0.347-0.403$ & $0.354-0.385$ \\
SDL/SL & $0.082-0.101$ & $0.089-0.092$ & $0.085-0.089$ & $0.085-0.095$ \\
LDL/SL & $0.149-0.179$ & $0.153-0.179$ & $0.141-0.157$ & $0.150-0.185$ \\
NL/SL & $0.104-0.110$ & $0.100-0.110$ & $0.102-0.109$ & $0.107-0.113$ \\
$\mathrm{ML} / \mathrm{SL}$ & $0.212-0.216$ & $0.196-0.220$ & $0.208-0.212$ & $0.219-0.227$ \\
$\mathrm{ED} / \mathrm{SL}$ & $0.077-0.105$ & $0.088-0.104$ & $0.066-0.077$ & $0.082-0.110$ \\
$\mathrm{PBL} / \mathrm{SL}$ & $0.139-0.142$ & $0.141-0.155$ & $0.149-0.163$ & $0.145-0.155$ \\
$\mathrm{CPH} / \mathrm{SL}$ & $0.095-0.117$ & $0.081-0.108$ & $0.087-0.107$ & $0.097-0.108$ \\
$\mathrm{CPL} / \mathrm{SL}$ & $0.080-0.086$ & $0.078-0.098$ & $0.066-0.083$ & $0.080-0.082$ \\
$\mathrm{ABL} / \mathrm{SL}$ & $0.150-0.176$ & $0.149-0.176$ & $0.143-0.152$ & $0.149-0.182$ \\
$\mathrm{LAL} / \mathrm{SL}$ & $0.139-0.193$ & $0.136-0.195$ & $0.149-0.151$ & $0.140-0.201$ \\
VSL/SL & $0.137-0.153$ & $0.146-0.160$ & $0.144-0.149$ & $0.135-0.157$ \\
STL/SL & $0.078-0.099$ & $0.088-0.104$ & $0.066-0.077$ & $0.085-0.102$ \\
\hline
\end{tabular}

Standard length (SL), body height (BL), caudal peduncle height (CPH), caudal peduncle length (CPL), shortest dorsal fin spine length (SDL), longest dorsal fin spine length (LDL), snout length (NL), maxilla length (ML), eye diameter (ED), pectoral fin base length (PBL), ventral fin spine length (VSL), anal fin base length (ABL), longest anal fin spine length (LAL) and supraocular tentacle length (STL).

Table S5. - Ranges of selected morphometric variables of S. scrofa populations.

\begin{tabular}{lccc}
\hline $\begin{array}{l}\text { Morphometric } \\
\text { Variables }\end{array}$ & Çanakkale & Hatay & İzmir \\
\hline BL/SL & $0.310-0.329$ & $0.292-0.324$ & $0.312-0.338$ \\
SDL/SL & $0.092-0.094$ & $0.075-0.098$ & $0.089-0.091$ \\
LDL/SL & $0.178-0.204$ & $0.171-0.185$ & $0.170-0.187$ \\
POH/SL & $0.126-0.136$ & $0.123-0.125$ & $0.132-0.140$ \\
NL/SL & $0.092-0.131$ & $0.091-0.126$ & $0.127-0.129$ \\
$\mathrm{ML} / \mathrm{SL}$ & $0.226-0.228$ & $0.212-0.219$ & $0.210-0.234$ \\
$\mathrm{PBL} / \mathrm{SL}$ & $0.130-0.149$ & $0.133-0.137$ & $0.149-0.166$ \\
$\mathrm{CPH} / \mathrm{SL}$ & $0.088-0.109$ & $0.092-0.097$ & $0.102-0.109$ \\
$\mathrm{ABL} / \mathrm{SL}$ & $0.148-0.156$ & $0.145-0.153$ & $0.138-0.177$ \\
VSL/SL & $0.145-0.171$ & $0.127-0.163$ & $0.144-0.215$ \\
\hline
\end{tabular}

Standard length (SL), body height (BL), caudal peduncle height (CPH), shortest dorsal fin spine length (SDL), longest dorsal fin spine length (LDL), preorbital height (POH), snout length (NL), maxilla length (ML), pectoral fin base length (PBL), ventral fin spine length (VSL) and anal fin base length (ABL). 